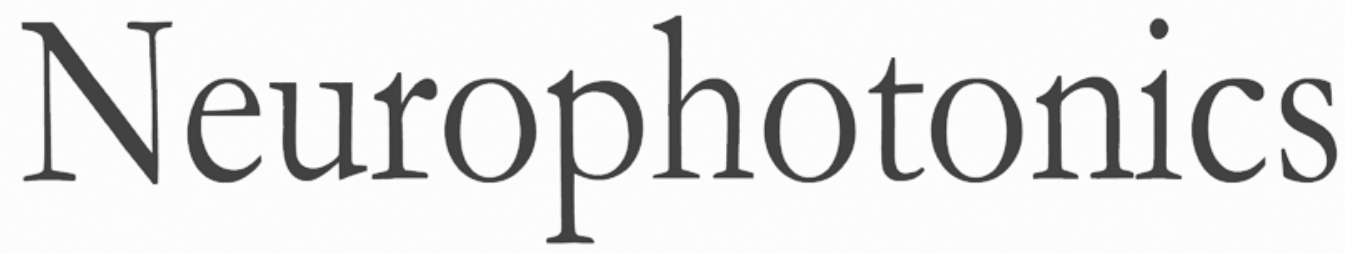

\title{
Alterations in neurovascular coupling following acute traumatic brain injury
}

Hyounguk Jang

Stanley Huang

Daniel X. Hammer

Lin Wang

Harmain Rafi

Meijun Ye

Cristin G. Welle

Jonathan A. N. Fisher 


\title{
Alterations in neurovascular coupling following acute traumatic brain injury
}

\author{
Hyounguk Jang, ${ }^{a, b}$ Stanley Huang, ${ }^{b}$ Daniel X. Hammer, ${ }^{b}$ Lin Wang, ${ }^{a}$ Harmain Rafi, ${ }^{a}$ Meijun Ye, ${ }^{b}$ \\ Cristin G. Welle, ${ }^{b, c}$ and Jonathan A. N. Fisher ${ }^{a, b, *}$ \\ ${ }^{a}$ New York Medical College, Department of Physiology, Valhalla, New York, United States \\ bU.S. Food and Drug Administration, Division of Biomedical Physics, Silver Spring, Maryland, United States \\ 'University of Colorado Denver, Departments of Neurosurgery and Bioengineering, Aurora, Colorado, United States
}

\begin{abstract}
Following acute traumatic brain injury (TBI), timely transport to a hospital can significantly improve the prognosis for recovery. There is, however, a dearth of quantitative biomarkers for brain injury that can be rapidly acquired and interpreted in active, field environments in which TBIs are frequently incurred. We explored potential functional indicators for TBI that can be noninvasively obtained through portable detection modalities, namely optical and electrophysiological approaches. By combining diffuse correlation spectroscopy with colocalized electrophysiological measurements in a mouse model of TBI, we observed concomitant alterations in sensory-evoked cerebral blood flow (CBF) and electrical potentials following controlled cortical impact. Injury acutely reduced the peak amplitude of both electrophysiological and CBF responses, which mostly recovered to baseline values within $30 \mathrm{~min}$, and intertrial variability for these parameters was also acutely altered. Notably, the postinjury dynamics of the CBF overshoot and undershoot amplitudes differed significantly; whereas the amplitude of the initial peak of stimulus-evoked CBF recovered relatively rapidly, the ensuing undershoot did not appear to recover within $30 \mathrm{~min}$ of injury. Additionally, acute injury induced apparent low-frequency oscillatory behavior in $\mathrm{CBF}(<1 \mathrm{~Hz})$. Histological assessment indicated that these physiological alterations were not associated with any major, persisting anatomical changes. Several time-domain features of the blood flow and electrophysiological responses showed strong correlations in recovery kinetics. Overall, our results reveal an array of stereotyped, injury-induced alterations in electrophysiological and hemodynamic responses that can be rapidly obtained using a combination of portable detection techniques. ๑ The Authors. Published by SPIE under a Creative Commons Attribution 3.0 Unported License. Distribution or reproduction of this work in whole or in part requires full attribution of the original publication, including its DOI. [DOI: 10.1117/1.NPh.4.4.045007]
\end{abstract}

Keywords: brain injury; diffuse correlation spectroscopy; neurophotonics; neurophysiology.

Paper 17110R received Aug. 9, 2017; accepted for publication Nov. 28, 2017; published online Dec. 23, 2017.

\section{Introduction}

In the United States, each year there are over 1.5 million traumatic brain injuries (TBIs), resulting in 50,000 deaths. ${ }^{1}$ TBI induces temporary or permanent impairment of cognition, physical function, and psychosocial behavior. ${ }^{2}$ TBI severity ranges widely depending on the nature of the injury. The majority of these injuries are classified as "mild" TBI (mTBI), which are challenging to diagnose and track because quantitative biomarkers for mTBI are lacking. In fact, one of the defining characteristics of mTBI is that it cannot be validated through standard methods of clinical imaging. ${ }^{3}$ Given the difficulty of rapid detection, mTBI poses a particular challenge to public health because repeated injuries such as concussions have a cumulative effect on brain health. ${ }^{4-6}$

Among a wide spectrum of TBI sequelae, sensory and cognitive deficits are some of the most common. ${ }^{7}$ A difficulty in assessing these deficits is that they are frequently mutually confounding. For example, auditory dysfunction following injury includes both peripheral deficits, e.g., increased hearing thresholds, as well as difficulties with more sophisticated auditory tasks, such as discriminating sounds in noisy environments or

*Address all correspondence to: Jonathan A. N. Fisher, E-mail: fisherworks@ gmail.com comprehending speech. ${ }^{8}$ Visual deficits following TBI are similarly complex; the most common complaints, for example, are accommodative deficiencies, ${ }^{9}$ which include blurred vision, headache, motion sickness, or loss of concentration during visual task performance. ${ }^{10}$ While auditory and visual performance may be difficult to dissociate from cognitive deficits, olfaction, which does not involve significant feedback with subcortical structures, is also impacted by TBI, and the degree of anosmia is correlated with the severity of injury. ${ }^{11}$

The integrity of sensory systems can be probed electrophysiologically via evoked potentials (EPs). EPs can be measured noninvasively and comprise a series of positive and negative voltage deflections, which reflect the afferent relay and processing of sensory information. Auditory-, visual-, and somatosensory-evoked potentials (SSEPs) are routinely used in multiple clinical contexts to aid neurological assessment of TBI and to monitor functional recovery over time. ${ }^{12}$ Sensory-evoked neural activity can also be inferred based on cerebral hemodynamic responses, including changes in cerebral blood flow $(\triangle \mathrm{CBF})$, volume, and oxygenation that are evoked by brief sensory stimuli. Damage to any portion of the cerebral vasculature fundamentally alters the ability of the network to supply neurons with energy. ${ }^{13}$ In fact, blockage of even single capillaries can cause larger-scale changes in blood flow ${ }^{14}$ and may result in microvascular ischemia. ${ }^{15}$ Repercussions of these injuries, 
even if mild, may continue to progress following the primary trauma, ultimately leading to more global sequelae.

We explored the possibility of deriving innovative indicators for mTBI based on intrinsic correlations between hemodynamic and neuronal activity. Specifically, we investigated signals that can be measured rapidly and using portable technology, which is a prerequisite for expedited assessment. To noninvasively monitor sensory-evoked hemodynamics, we used diffuse correlation spectroscopy (DCS) that takes advantage of the dynamic scattering properties of red blood cells to directly measure cerebral blood flow (CBF). DCS is particularly sensitive to flow in the cortical microvasculature due to the high absorption (and thus low probability of photon escape) in larger blood vessels. ${ }^{16}$ DCS has been used to measure functional hemodynamics associated with sensory stimuli and motor tasks ${ }^{17,18}$ and has been used to track baseline CBF following brain trauma. ${ }^{19}$ We supplemented our ongoing optical recordings of the sensory-evoked $\triangle \mathrm{CBF}$ with concomitant measurements of SSEPs and applied this multimodal approach to a mouse model of TBI that employed controlled cortical impact (CCI) as the source of primary injury. ${ }^{20-22}$ The ability to noninvasively monitor both aspects of neural response enabled us to obtain the first detailed, in vivo portrait of the effects of acute injury on sensory processing in the brain.

\section{Materials and Methods}

\subsection{Surgical Procedures}

All animal experiments were performed in accordance with the guidelines of the White Oak Institutional Animal Care and Use Committee. Optical and electrophysiological measurements were performed on 10 male C57BL/6J mice (12 to 24 weeks). Anesthesia was induced by an initial exposure to $4 \%$ isoflurane (vaporized in medical grade compressed oxygen) for $<30 \mathrm{~s}$. Animals were additionally administered an injection of xylazine $(18 \mathrm{mg} / \mathrm{kg}, \mathrm{IP})$ to provide a stable plane of anesthesia at low isoflurane concentrations $(0.1 \%$ to $0.25 \%)$ for the remainder of the experimental session, which typically lasted 3 to $6 \mathrm{~h}$. Maintenance doses of xylazine $(6 \mathrm{mg} / \mathrm{kg})$ were administered once every $\sim 2.5 \mathrm{~h}$. Following initial anesthesia induction, animals were positioned in a stereotaxic apparatus (David Kopf Instruments, California). Their body temperature was measured and maintained at $37^{\circ} \mathrm{C}$ with a closed-loop temperature-controlled heating pad (Model TC1000, CWE). Respiratory rate was also monitored and maintained at $\sim 100$ breaths / min during the surgical and experimental procedures. Skin incisions were infused with lidocaine, and the eyes were covered with ointment (Lacri-Lube) to prevent drying. A midline sagittal incision was made in the skin, which exposed the coronal and lambdoid sutures on the skull. The intersection of these sutures with the midline (i.e., bregma and lambda) served as landmarks for recording locations and was also used as a guide when drilling burr holes. The area of the skull under the probe was cleaned with $70 \%$ ethanol, and the optical probe was secured to the skull by cyanoacrylate glue (Loctite 454, Hankel, Australia) [Fig. 1(a)]. Burr holes (diameter: $\sim 0.5 \mathrm{~mm}$ ) were made for placement of two silver wire electrodes: a recording electrode, placed 2.5-mm lateral and 1-mm posterior to bregma, and a reference electrode, placed 1-mm lateral and 1-mm posterior to lambda. The recording electrode was embedded within the optical probe tip, and the reference electrode was secured into position with Kwik-Sil adhesive
(World Precision Instruments, Florida). A ground needle electrode was placed subcutaneously on the back of the animal.

Although epidural measurements of SSEPs are invasive, we have previously compared the effects of injury on SSEPs measured both epidurally and epidermally. ${ }^{23,24}$ In mouse experiments, SSEP waveforms measured using both approaches differ only in signal-to-noise ratio, which is roughly an order of magnitude higher when measured epidurally. In this study, the use of epidural recordings permitted us to rapidly acquire clear SSEPs at a high sampling rate following injury owing to the fact that less time was needed to be spent averaging, compared with epidermal recordings. This enhanced the temporal resolution with which injury-induced changes could be tracked.

\subsection{Sensory Stimuli and Electrophysiological Recordings}

SSEPs were recorded in single-ended configuration (RZ5D processor, PZ2 preamplifier, ZC16 headstage, Tucker-Davis Technologies, Florida) with a shared common reference electrode, at a sampling frequency of $3 \mathrm{kHz}$. Optical measurements of $\triangle \mathrm{CBF}$ were performed concurrently, driven by a separate computer. The median nerve contralateral to the recording locations was stimulated via a pair of 27 -gauge stainless-steel needles inserted subcutaneously into the forelimb of the animal. The electrical stimulus consisted of a train of 12 current pulses generated by a constant current stimulator (DS7A, Digitimer) (amplitude $4 \mathrm{~mA}$, frequency $3 \mathrm{~Hz}$, pulse duration $200 \mu \mathrm{s}$, and total pulse-train duration $4 \mathrm{~s}$ ). Individual measurements of sensory-evoked $\triangle \mathrm{CBF}$ were separated by $45 \mathrm{~s}$, which we empirically found to be the minimum intertrial duration that did not elicit alterations in the steady-state CBF. An overview of the acquisition and stimulus timing is shown in Fig. 1(b). The major peaks associated with the SSEP waveform were identified with MATLAB ${ }^{\circledR}$ as maxima and minima within boundaries set using published values as a reference ${ }^{23,25,26}$ as follows: P1 corresponded to the time of maximum voltage within the window of 15 to $30 \mathrm{~ms}$, N1 corresponded to the time of minimum voltage within the window of 21 to $50 \mathrm{~ms}$, and $\mathrm{P} 2$ corresponded to the time of maximum voltage at times later than the identified N1 time.

\subsection{Controlled Cortical Impact}

CCI is a well-established and highly reproducible brain injury model. ${ }^{20,21,27-30}$ Closed-skull impact was delivered with an Impact One ${ }^{\mathrm{TM}}$ stereotaxic impactor (Leica Microsystems). Using a 5-mm-diameter metal impact tip, we used the following settings: velocity $4 \mathrm{~m} / \mathrm{s}$, dwell time $100 \mathrm{~ms}$, and impact depth $0.6 \mathrm{~mm}$. Strike velocities $>5 \mathrm{~m} / \mathrm{s}$ and/or strike depths $>$ $0.8 \mathrm{~mm}$ resulted in skull fracture. Additionally, impactor tips with smaller diameter tended to cause fractures. The metal impactor tip was positioned over the exposed skull $\sim$-mm anterior and $\sim 1-\mathrm{mm}$ lateral of bregma, contralateral to the optical measurement.

\subsection{Optical Measurement of Cerebral Blood Flow}

The DCS signal can be used to measure blood flow by using speckle correlation techniques. The basic theory underlying DCS has been extensively described in previous publications. ${ }^{16,31}$ Briefly, when laser light migrates through tissue, the emerging intensity pattern, called a speckle pattern, is composed of bright 


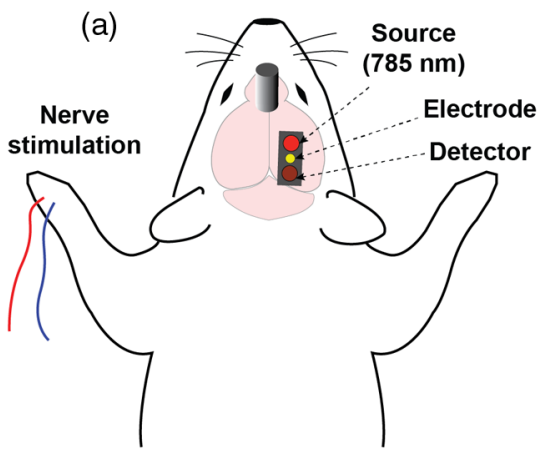

(c)

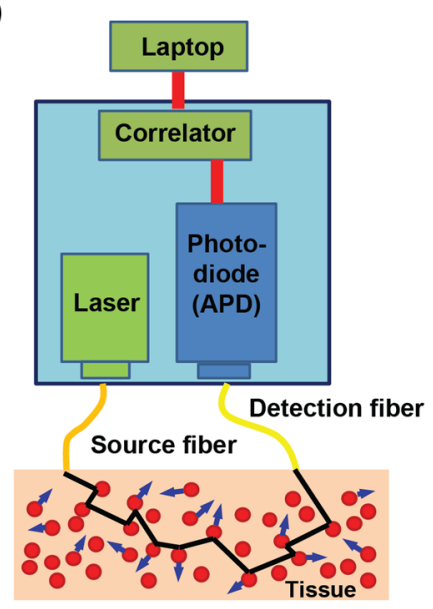

(b)

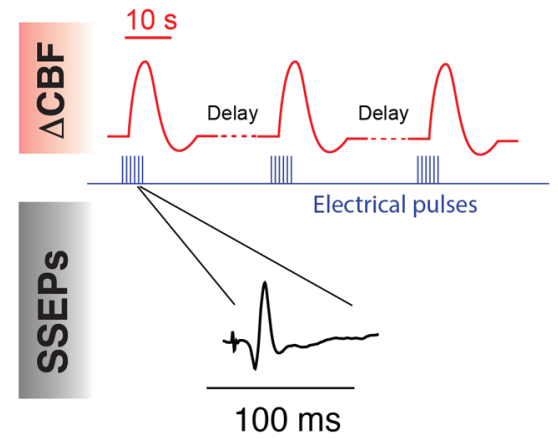

(d)
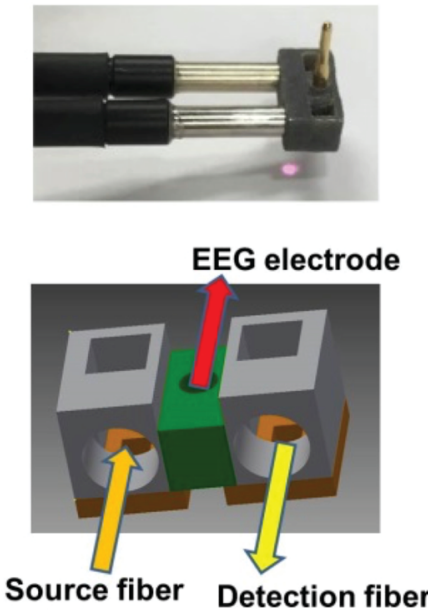

Fig. 1 Experimental design and measurement apparatus. (a) Schematic of the recording and stimulation configuration. A DCS probe tip containing optical fibers is placed on the region of the skull above the primary somatosensory cortex, at a location coinciding with the forepaw's representation. A recording EEG electrode is inserted through the center of the probe. Pulses of electrical current are delivered to the contralateral limb with respect to the optical and electrophysiological measurements. Closed-skull impact is delivered by a 5-mm impactor tip $\sim 4-\mathrm{mm}$ anterior and $\sim 1-\mathrm{mm}$ lateral of bregma contralateral to the optical measurement (see Sec. 2). (b) Overview of synchronization between stimulus, measurements of $\mathrm{CBF}$, and SSEPs. The red traces depict simulated hemodynamic signals, which are broad compared with electrophysiological potentials (note the time scale difference in the insets). The blue pulses indicate electrical stimuli delivered to the median nerve. (c) Block diagram of the DCS device used in this study. (d) Photograph and schematic of the 3-D-printed DCS probe tip. Light is delivered and collected from the tissue through fiber optics that are coupled to the skin with microprisms.

and dark spots, which are caused by constructive and destructive interference of photons that traverse different path lengths. The intensity fluctuations of a single region, which are caused by interactions of scattered light with moving particles (i.e., red blood cells), can be utilized to extract information about blood flow.

A block diagram of our DCS recording system is shown in Fig. 1(c). It consists of a long coherence length continuous-wave near-infrared laser (785 nm, CrystaLaser, Nevada), a photoncounting avalanche photodiode (APD) (SPCM-AQRH-12-FC, Excelitas, Quebec, Canada), and an autocorrelator signal processing board (Ref. 32, New Jersey). NIR excitation light was delivered to the brain with a multimode optical fiber (200- $\mu \mathrm{m}$ core diameter, Thorlabs, New Jersey), and the scattered light was detected with a single-mode fiber $(5-\mu \mathrm{m}$ core diameter) connected to the APD. The source and detection fibers were separated by $5 \mathrm{~mm}$. The APD signal was sent to the correlator board, which computed the intensity of the autocorrelation function. The correlation board streams output signals continuously via USB to a laptop PC for further data analysis.
The probe that coupled the optical fibers and recording electrode to the head was fabricated from semiflexible acrylate polymer using a three-dimensional (3-D) printer (Objet 260 Connex 3 printer, Stratasys, Minnesota). Embedded microprisms directed light from the fibers down to the head. The recording electrode was situated at the midpoint between the DCS optical source-detector separation. The midpoint of the probe [indicated in green in Fig. 1(d)] was thinner and permitted the probe to bend and conform to the curvature of the mouse's skull, providing tight contact to the surface. The probe was positioned on a relatively flat region of intact skull above the forelimb's representation in primary somatosensory cortex, $2.5-\mathrm{mm}$ lateral of the midline at the same coordinate as bregma on the rostrocaudal axis. ${ }^{33}$

\subsection{Modeling the Hemodynamic Response Function}

To extract quantitative features from the sensory-evoked $\Delta \mathrm{CBF}$, we fit the observed waveform to a canonical hemodynamic response function (HRF) that uses two-gamma density functions to approximate the hemodynamic response [Fig. $2(\mathrm{a})],{ }^{34}$ i.e., 
(a)

Cerebral blood flow

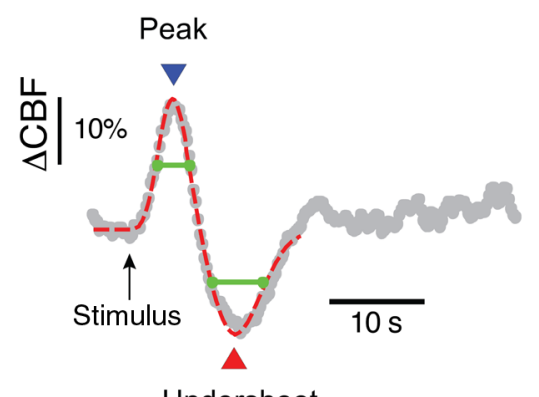

(b) Evoked potential

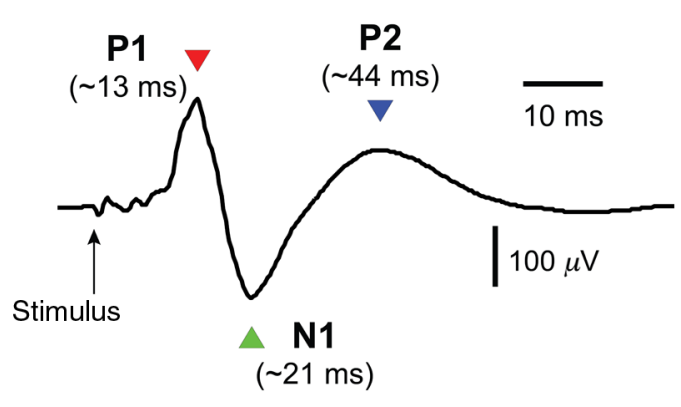

Fig. 2 Somatosensory-evoked hemodynamic and electrophysiological responses. (a) Optically measured changes in relative CBF following electrical stimulation of the median nerve. The gray trace represents the average of 10 trials in one experiment; blood flow values are normalized to the average of CBF values recorded in $4 \mathrm{~s}$ prior to the stimulus onset, which is indicated by the arrow. The dotted red line that is superimposed on the trace represents a fit to the observed hemodynamic response using a conventional HRF model (two-gamma functions), as described in Sec. 2. (b) SSEP recorded concurrently during acquisition of the optical data depicted in (a). The prominent peaks P1, N1, and P2 occur at temporal latencies that are well-established in the literature. The trace represents the average obtained from the same 10 trials in (a); however, for each trial, there are 12 SSEPs recorded in response to individual stimulus pulses ( $4 \mathrm{~s}$ of pulses delivered at $3 \mathrm{~Hz}$ ); thus, the trace represents the average of 120 SSEPs. The green lines represent the widths of positive and negative peaks, measured at half of the maximum/ minimum values. Note that the timescale in (a) is roughly $1000 \times$ longer than in (b).

$\operatorname{HRF}(t)=A\left[\frac{t^{\alpha_{1}-1} \beta_{1}^{\alpha_{1}} e^{-\beta_{1} t}}{\Gamma\left(\alpha_{1}\right)}-c \frac{t^{\alpha_{2}-1} \beta_{2}^{\alpha_{2}} e^{-\beta_{2} t}}{\Gamma\left(\alpha_{2}\right)}\right]$

where $\Gamma$ represents the gamma function. $A, c, \alpha_{1}, \alpha_{2}, \beta_{1}$, and $\beta_{2}$ are the fitting coefficients, which were fit using the LevenbergMarquardt algorithm in MATLAB ${ }^{\circledR}$. Here, $A$ controls the amplitude, $c$ determines the ratio of the response to undershoot, and $\alpha_{1}, \alpha_{2}, \beta_{1}$, and $\beta_{2}$ control the shape of the fit function. We obtained four major features, which are the amplitude and temporal delay (relative to stimulus onset) of the $\triangle \mathrm{CBF}$ onset (initial peak) and offset (return to baseline), to quantitatively describe the blood flow response based on the fitted curve. Although this model is typically applied to aspects of the hemodynamic response other than $\triangle \mathrm{CBF}$, namely the blood-oxygenlevel-dependent signal, the model is generic in that it can accommodate a bimodal onset/offset function. For instance, the model can just as easily fit a response with no apparent undershoot and can capture waveform attributes such as a highly skewed peak. For quantifying trends in the CBF waveforms, the peak and undershoot were identified based on the fit to the dual gamma distribution model, and a criterion of $R^{2}>0.9$ was used in the fitting process.

\subsection{Data Quality Criteria for Inclusion}

Animals varied in the magnitude of noise associated with CBF and electrophysiological measurements. Sources of variability included optical coupling efficiency, electrode impedances, and physiological noise, among others. To best quantify injury-related changes, we only included data from experiments in which preinjury optical and electrophysiological noise levels were low enough to permit the observation of clear stimulusevoked CBF changes and SSEPs. Quantitatively, our data inclusion criterion for optical measurements was that the initial CBF peak had an amplitude that exceeded twice the standard deviation (SD) of the baseline signal (i.e., $z$-score $>2$ ).

\subsection{Time-Frequency Analysis of the Hemodynamic Response Function}

In the interest of parsing apparent oscillatory behavior associated with postinjury modifications in the hemodynamic response following the main positive and negative peaks, we performed spectrotemporal analysis on the CBF data throughout the entire duration of six experiments. Briefly, the continuous DCS data were divided into six 5-min epochs, and analysis of the evoked responses within those epochs was used to inform single "frames." We utilized time-frequency transform functions from the MATLAB ${ }^{\circledR}$ toolbox EEGLAB ${ }^{35}$ to produce spectrograms that quantified power as well as intertrial coherence (ITC), which indicates the degree of phase locking in the observed $\triangle \mathrm{CBF}$. Note that for each data point in Figs. 9 and 10 , the spectrograms were based on only the trials within the 5-min epochs; although a larger sample size generally improves signal-to-noise, in this case because the $\triangle \mathrm{CBF}$ was dynamically changing in time following injury, there was a trade-off between signal-to-noise and the temporal resolution with which the time evolution of power and ITC could be quantified. We empirically converged on 5-min epochs as a compromise based on observations from the continuous $\mathrm{CBF}$ that indicated that recovery generally occurred on a timescale slower than 5 min following a rapid decrement due to $\mathrm{CCI}$.

\subsection{Histological Procedures}

We assessed the effects of CCI on the brain by performing vital staining and immunohistological analysis. Two mice were subjected to CCI using the same parameters as all of the functional monitoring experiments (velocity: $4 \mathrm{~m} / \mathrm{s}$; dwell time: $100 \mathrm{~ms}$; and impact depth: $0.6 \mathrm{~mm}$ ). Another two animals were subjected to CCI of higher severity (impact depth was increased to $1.1 \mathrm{~mm}$ ), and an additional two sham animals received no impact yet were positioned in the stereotaxic apparatus and the cortical impactor was fixed above the animal's head. Animals were permitted to recover following the procedure. 
$24 \mathrm{~h}$ after CCI or sham treatment, animals were anesthetized with sodium pentobarbital $(100 \mathrm{mg} / \mathrm{kg}$, i.p.) and perfused transcardially with saline and then formalin. Mice were then decapitated and the brain removed and postfixed in formalin overnight. $50-\mu \mathrm{m}$ coronal brain sections were acquired with a vibrating microtome at anteroposterior (AP) levels spanning bregma $+2 \mathrm{~mm}$ through $-2 \mathrm{~mm}$. For glial fibrillary acidic protein (GFAP) immunohistological analysis, floating slices were incubated overnight with monoclonal antimouse GFAP antibody (1:500 dilution, Invitrogen). The slices were then incubated with Peroxidase-conjugated AffiniPure Goat Antirat IgG $(\mathrm{H}+\mathrm{L})$ antibody (1:100 dilution, Jackson ImmunoResearch Laboratories) and stained with a 3,3-diaminobenzidine horseradish peroxidase substrate kit (Vector Laboratories), then mounted onto gelatin subbed slides and coverslipped. For Nissl staining, sections from the same brain, yet AP $+50 \mu \mathrm{m}$ to the locations at which slices for GFAP analysis, were mounted and dried on gelatin subbed slides. They were then immersed in a heated solution of $0.1 \%$ cresyl violet $(\mathrm{CV})$ acetate (Electron Microscopy Sciences) for $4 \mathrm{~min}$ and dehydrated in alcohol. Slides were then cover slipped with Permount (Fisher Scientific) and dried before imaging.

\section{Results}

\subsection{Concurrent Measurements of Sensory-Evoked Cerebral Hemodynamics and SSEPS}

Using the DCS component of our multimodal apparatus, we recorded sensory-evoked changes in $\mathrm{CBF}$ concurrently with electrophysiological measurements of SSEPs. Figure 2(a) shows an example of the preinjury CBF response. Averaged over 10 animals, the $\triangle \mathrm{CBF}$ displayed an initial increase in blood flow of $14 \% \pm 4.8 \%$ (mean \pm SD) that peaked at $9 \pm 0.3 \mathrm{~s}$, followed by a subsequent undershoot of magnitude $-12 \% \pm 2.5 \%$, which reached a negative peak $17 \pm 1.1 \mathrm{~s}$ after the stimulus ( $12 \pm 1.1 \mathrm{~s}$ after the first peak). CBF returned to baseline values roughly $10 \mathrm{~s}$ following maximum undershoot. In terms of electrophysiological recordings, SSEPs displayed well-defined peaks, most prominently a positive deflection (P1) at $\sim 12-$ ms poststimulus, a negative peak (N1) at $\sim 21 \mathrm{~ms}$, and a broader positive peak at $\sim 44 \mathrm{~ms}$ (P2) [Fig. 2(b)].

\subsection{TBI Acutely Alters Hemodynamic and Electrophysiological Functional Responses}

Cortical impact acutely reduced the amplitude and altered the waveforms of sensory-evoked hemodynamic and electrophysiological responses (Fig. 3). Within the first $5 \mathrm{~min}$ following impact, the $\triangle \mathrm{CBF}$ response peak decreased by $\sim 50 \%$, and the undershoot amplitude decreased as well, though to a lesser extent ( $\sim 20 \%$ to $40 \%)$. The peak latencies additionally displayed changes on the order of $\sim 10 \%$, though with opposite trends; the initial increase in CBF peaked $\sim 1$ s earlier than preinjury, and the undershoot peaked $\sim 1 \mathrm{~s}$ later than in the preinjury case. The alterations in these parameters, based on the results of 10 mice, are summarized in Fig. 4. The peak-topeak amplitude recovered to $\sim 80 \%$ of its baseline value within $30 \mathrm{~min}$, and during this period, the shifts in peak latencies resolved completely. It should be noted that despite being more sensitive to injury than other features of the $\triangle \mathrm{CBF}$ waveform, the initial peak also recovered at a relatively fast rate, and within 5 to $10 \mathrm{~min}$ following injury, the peak amplitude had

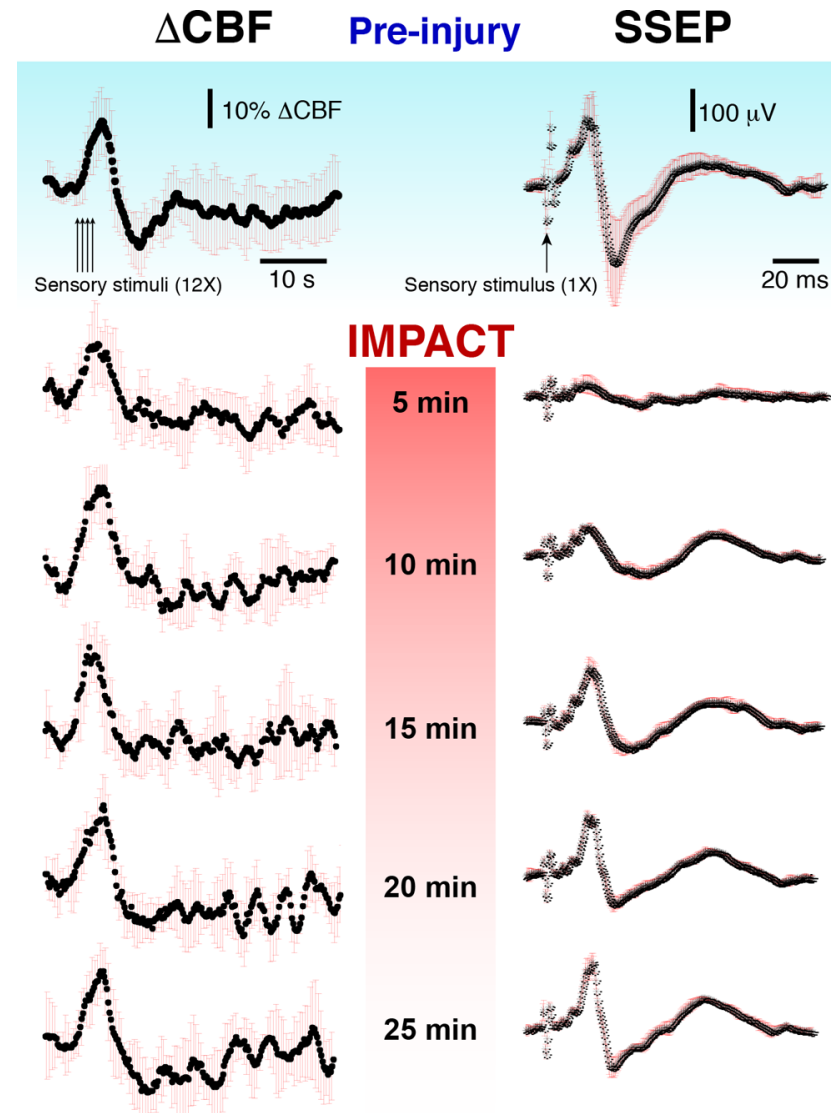

Fig. 3 Sensory-evoked blood flow responses from forepaw stimulation for a representative experiment pre- and postinjury. The DCBF and SSEP traces shown here represent the average of multiple stimulations (average of 10 trials preinjury and 5 trials for each time point postinjury). The black line is the average, and the red error bars represent a SD.

essentially recovered completely. In contrast, the undershoot alterations following injury, though more difficult to quantify because of the variability in that portion of the waveform post-CCI, displayed no recovery trend within the same time period.

SSEPs displayed similarly profound alterations within the first $5 \mathrm{~min}$ after CCI. As shown in Fig. 5, P1 fell to 40\% of its preinjury amplitude; however, N1 and P2 exhibited greater reductions of $81 \%$ and $78 \%$, respectively. CCI additionally altered the peak latencies. Although the P1 latency increased by less than $\sim 14 \%, \mathrm{~N} 1$ and $\mathrm{P} 2$ both increased by up to $\sim 13 \%$ and $\sim 50 \%$. In general, P1 appeared to be the least sensitive to injury, in terms of both amplitude and latency. In the ensuing $30 \mathrm{~min}$, the peak latencies and amplitudes largely—although not entirely-returned to their baseline values, yet at differing rates. The amplitude of $\mathrm{P} 2$, for example, recovered more rapidly than $\mathrm{N} 1$ or P1 in the first $15 \mathrm{~min}$. Overall, however, following CCI, the initial decrease in SSEP amplitude (peak-to-peak, $\mathrm{N} 1$ to $\mathrm{P} 2$ ) was significantly more profound than the drop in CBF. For example, SSEP peak-to-peak amplitude was reduced by nearly $75 \%$, while CBF peak-to-peak dropped by only $\sim 45 \%$.

In addition to alterations in average amplitude and latency of SSEP and CBF features, injury acutely increased their trial-to-trial variability of these parameters (Fig. 6). In our time-domain measurements, we quantified this variability in 

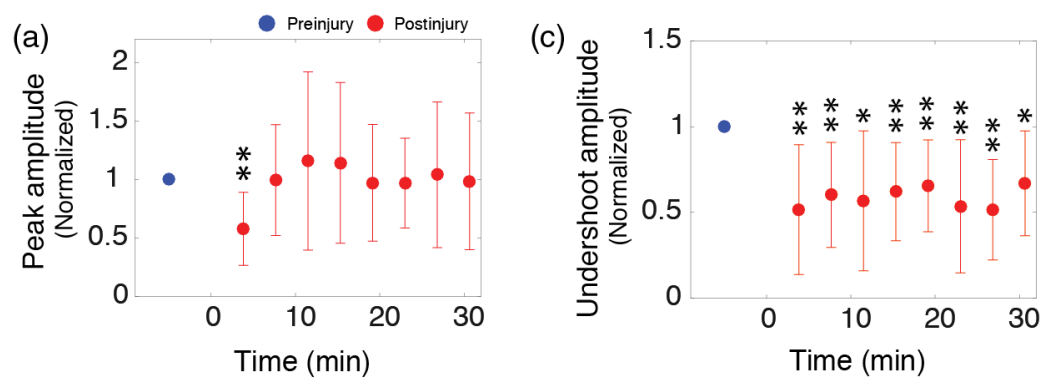

(e) $\quad \Delta$ CBF (Sham)
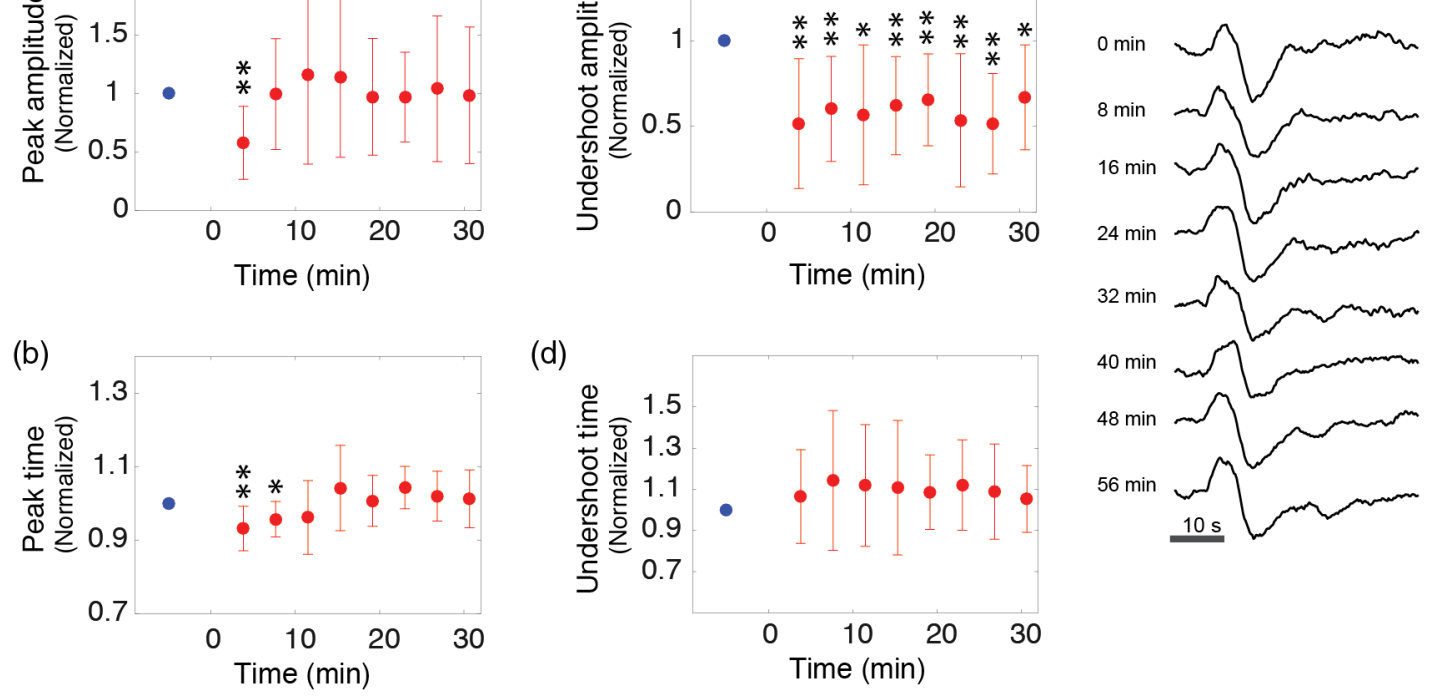

Fig. 4 (a)-(d) The grand average summary of the effects of $\mathrm{CCl}$ on the amplitude and latency of prominent time-domain features of the $\triangle \mathrm{CBF}$ waveform. The red dots represent the average values over all animals $(n=10)$. Values are normalized to the baseline value (blue dot). The time of impact is designated here as $t=0 \mathrm{~s}$. Error bars represent SD. Significant changes relative to baseline are indicated by asterisks, which refer to $P$-values obtained from a two-tailed, paired student's $t$-test $(*=P<0.05$, $* *=P<0.01$ ). (e) A montage of sensory-evoked $\triangle \mathrm{CBF}$ waveforms taken repeatedly over the course of an hour in a sham experiment.
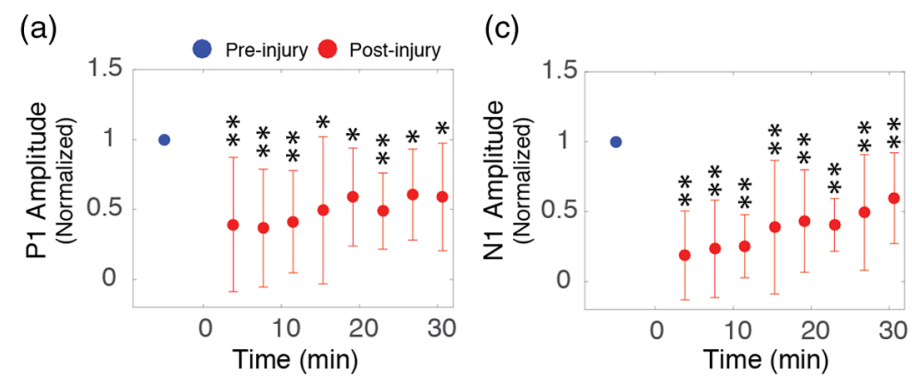

(e)
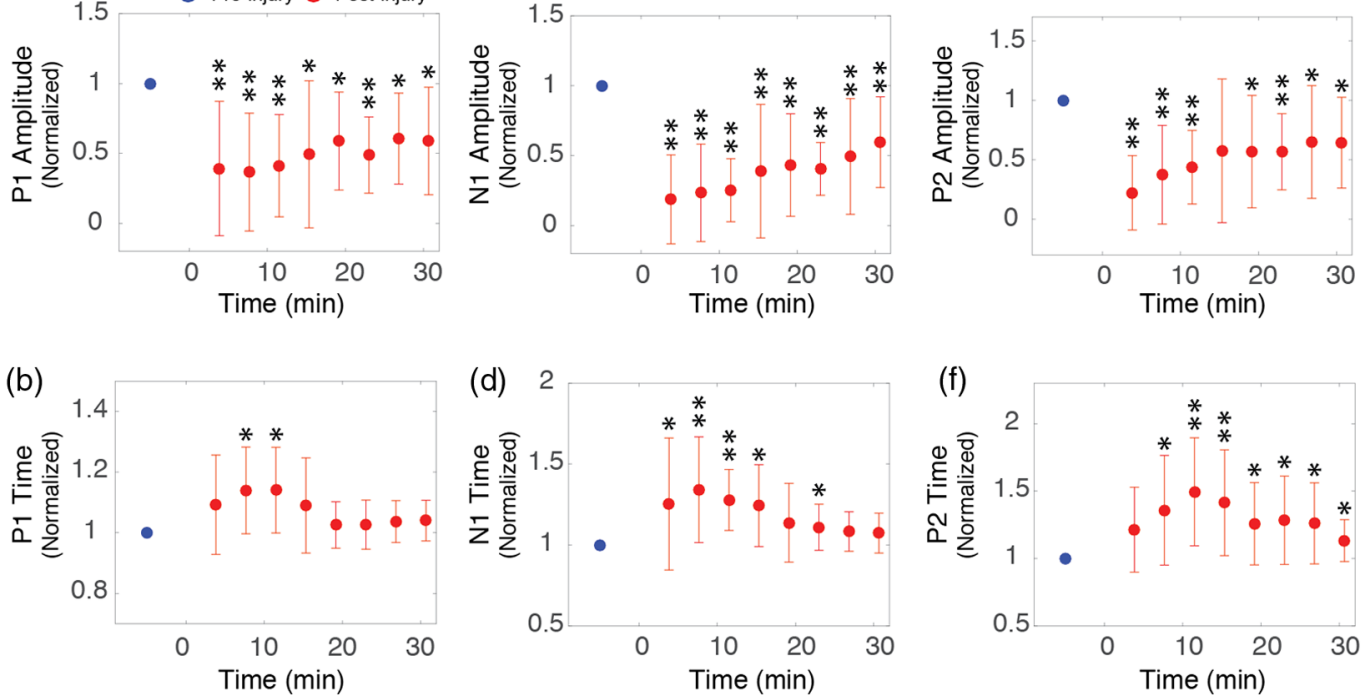

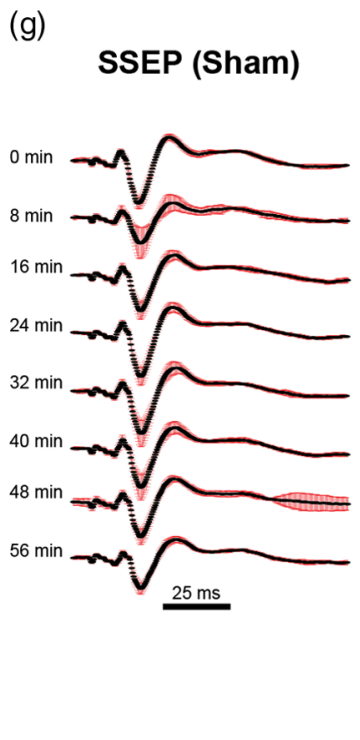

Fig. 5 (a)-(f) The grand average summary of the effects of $\mathrm{CCl}$ on major SSEP peaks. As in Fig. 4, red data points indicate postinjury averages over all animals $(n=10) \pm$ SD and are normalized to baseline values (blue data points). The time of impact is designated here as $t=0 \mathrm{~s}$, and significance relative to baseline is indicated by asterisks, as in Fig. 4. (g) A montage of SSEP waveforms taken repeatedly over the course of an hour in a sham experiment.

terms of the coefficient of variation, $C_{v}$, which is defined as the ratio of the SD to the mean value. In the first 5 min following CCI, $C_{v}$ increased by over $200 \%$ for all electrophysiological and blood flow parameters. Additionally, while the variability of other parameters returned to near baseline values within $10 \mathrm{~min}$ after injury, the CBF undershoot amplitude maintained an elevated variability over $30 \mathrm{~min}$ after injury, on average.

\subsection{Histological Assessment of the Effects of $\mathrm{CCl}$}

To assess the repercussions of our implementation of cortical impact on the brain, we explored both macroscopic, tissuelevel effects as well as GFAP expression in brains of animals that had been exposed to CCI (Figs. 7 and 8, respectively). We assessed effects at locations (1) close to the site of impact, which was anterior to the site of DCS and SSEP measurements, 

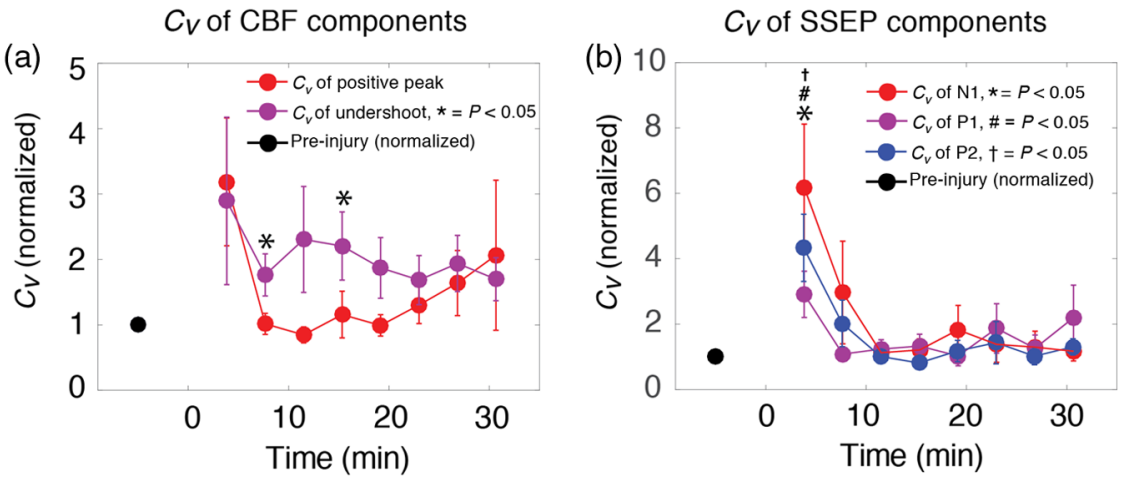

Fig. 6 Alterations in the variability of evoked responses following injury. (a) Dynamics of the $\triangle$ CBF variability are plotted over time, normalized to a baseline value (black dot). Injury was induced at 0 min in these plots. Variability is quantified here in terms of the coefficient of variation, $C_{v}$, defined as the ratio of the SD to the mean. $C_{v}$ is plotted for both the initial $\triangle \mathrm{CBF}$ peak (in red) and the subsequent undershoot (purple). (b) Evolution of $C_{V}$ for SSEP peaks N1 (red), P1 (purple), and P2 (blue), normalized to baseline values (black dot). Error bars represent standard error of the mean, and $P$-value indications are as described in the figure insets.

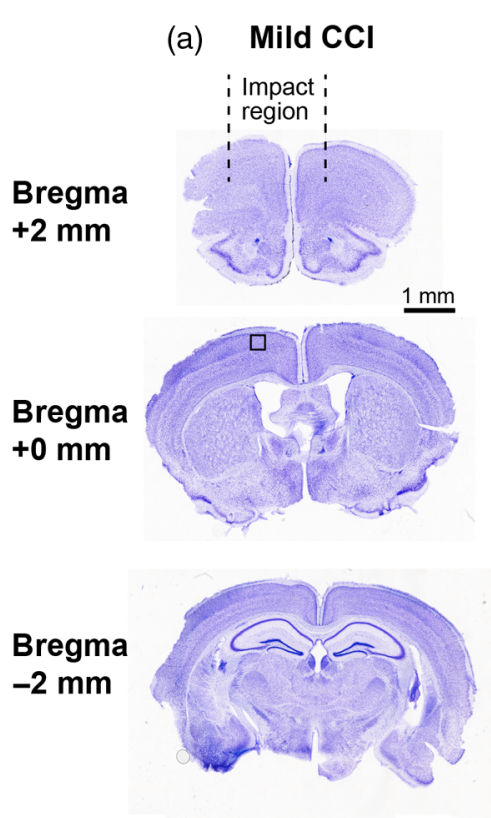

(d)

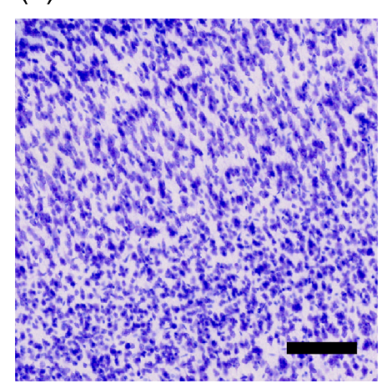

(b) Moderate $\mathrm{CCl}$

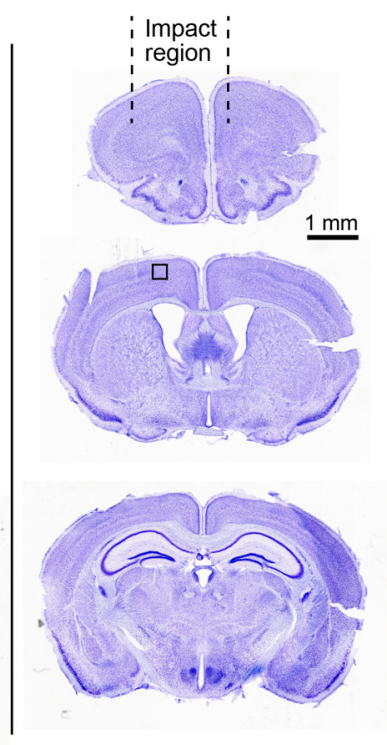

(e)

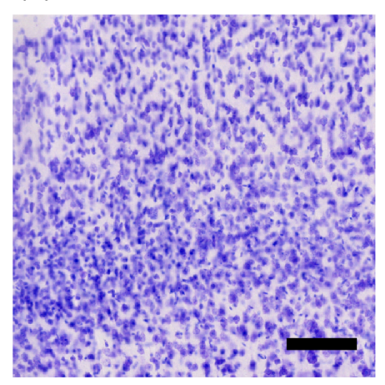

(c) Sham

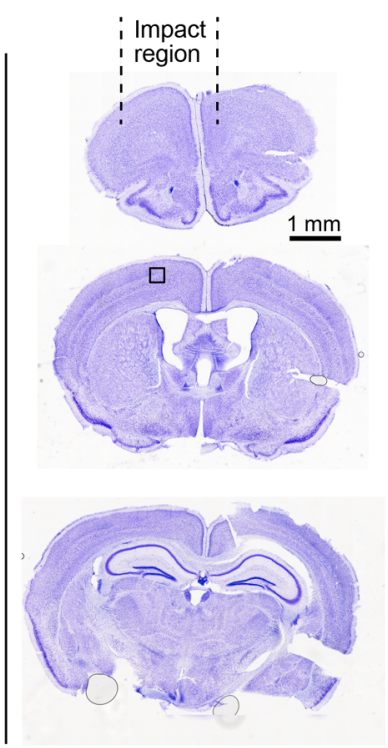

(f)

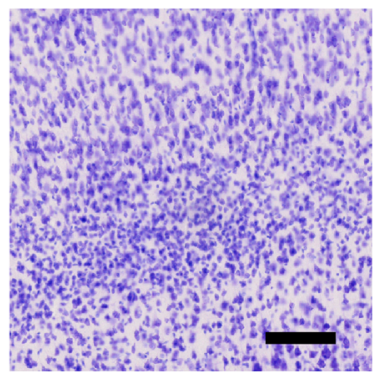

Fig. $7 \mathrm{CV}$ staining reveals negligible macroscopic anatomical damage $24 \mathrm{~h}$ after $\mathrm{CCl}$. Columns (a) and (b) depict CV-stained coronal brain sections from mice that received "mild" and "moderate" CCl treatment, respectively, and (c) depicts CV-stained sections from sham experiments. Here, "mild" $\mathrm{CCl}$ corresponds to the parameter settings employed for all of the functional data in this study (velocity: $4 \mathrm{~m} / \mathrm{s}$; dwell time: $100 \mathrm{~ms}$; impact depth: $0.6 \mathrm{~mm}$ ), while "moderate" corresponds to an increased impact depth $(1.1 \mathrm{~mm})$, with other parameters remaining the same. Slices from each condition are shown at locations on the AP axis corresponding to $+2 \mathrm{~mm}, 0 \mathrm{~mm}$, and $-2 \mathrm{~mm}$ relative to bregma. Square subregions that are indicated in the slices at bregma $+0 \mathrm{~mm}$ (middle row) are shown on an expanded scale (imaged at $40 \times$, scale bar $=100 \mu \mathrm{m})$ in (d) $-(\mathrm{f})$. 


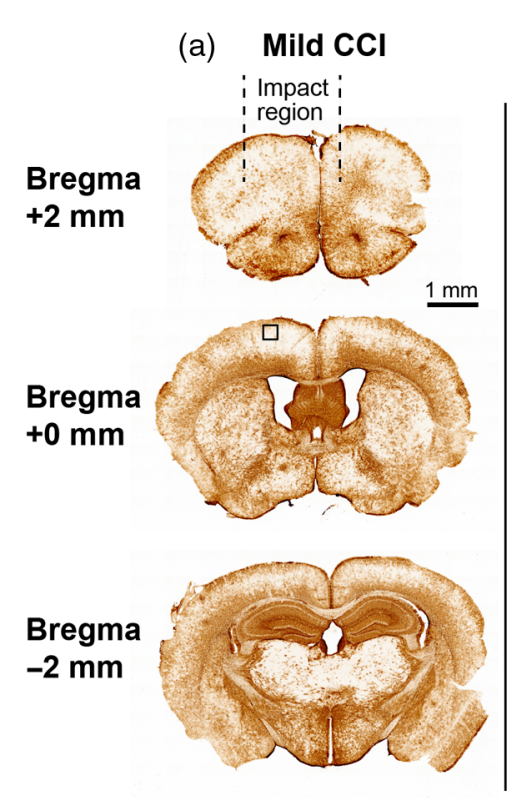

(d)

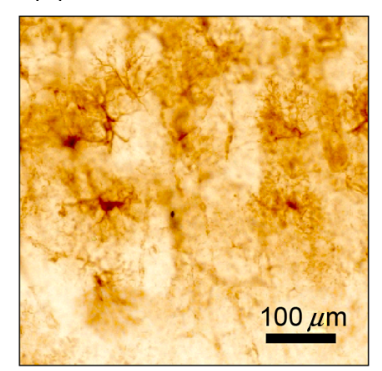

(b)

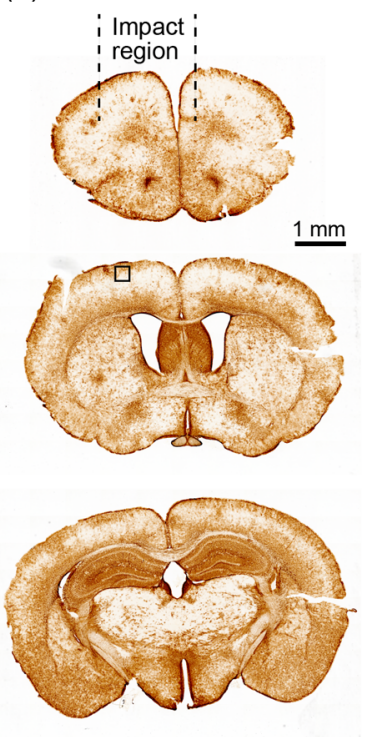

(e)

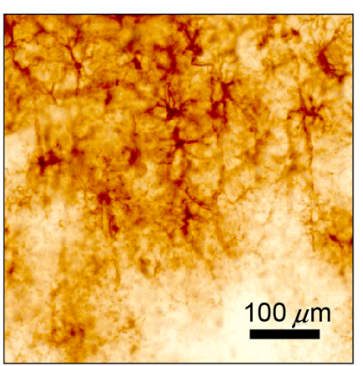

(c)

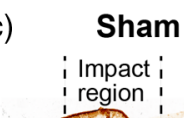
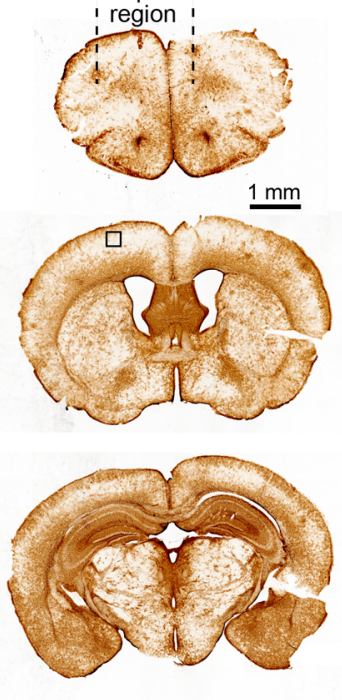

(f)

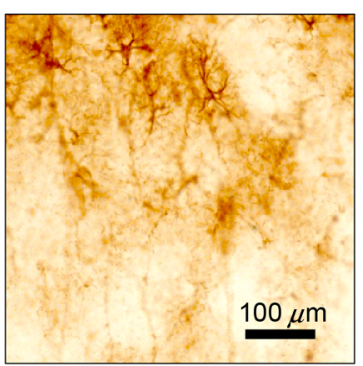

Fig. 8 GFAP immunoreactivity $24 \mathrm{~h}$ after $\mathrm{CCl}$ indicates mild astrogliosis, but no macroscopic damage. Following the layout of Fig. 7, columns (a) and (b) depict GFAP immunoreactivity in coronal slices from mice that received mild and moderate $\mathrm{CCl}$ treatment, respectively, and (c) depicts results from sham experiments. Definitions of mild and moderate are as described in the caption for Fig. 7. Mild CCl corresponds to the parameter settings employed for all of the functional data in this study. The small notches on the ventrolateral corners of the brain slices indicate the hemisphere contralateral to the central axis of the impactor tip. Subregions that are indicated in the slices at bregma $+0 \mathrm{~mm}$ (middle row) are shown on an expanded scale in (d)-(f).

(2) at the same AP location as the optical and electrophysiological measurements, and (3) at a location distal (posterior) to the site of impact.

Figure 7 shows CV Nissl staining of brain sections from animals that had been exposed to CCI. Figure 7(a) shows CVstained slices from an animal subjected to the same CCI parameters used during acquisition of all functional data (i.e., Figs. 3 6 and 9-11); we termed these settings "mild" for the sake of comparison with the histological results obtained following a slightly more intense impact (i.e., impact depth of $1.1 \mathrm{~mm}$ versus 0.8 ), which we termed "moderate." Compared with sham animals, CV staining revealed no evidence of significant alterations in tissue morphology, such as persisting edema, major hematoma, or direct lesions resulting from the CCI settings used throughout this study (two animals per condition). GFAP immunoreactivity (Fig. 8), however, revealed a proliferation of reactive astrocytes biased toward the hemisphere that was exposed to a greater area of the impactor tip (which was $5 \mathrm{~mm}$ in diameter). This astrogliosis effect was evident at the site on the AP axis where functional measurements were centered and was more pronounced at moderate CCI (impact depth $1.1 \mathrm{~mm})$ compared with mild $(0.8 \mathrm{~mm})$. Although GFAP immunoreactivity appeared to be more globally intense at the most distal region assessed (AP $-2 \mathrm{~mm}$ ), this is consistent with baseline expression patterns of GFAP, ${ }^{36}$ and there was no apparent difference relative to the sham condition.

\subsection{Spectrotemporal Analysis the Hemodynamic Response Function Following TBI}

Modulation of prominent aspects of the hemodynamic response such as peak amplitude and latency are straightforward to quantify because the waveforms can fit well to a conventional HRF model (as described in Sec. 2). However, injury also induced new temporal features into the CBF response. For example, in the $\sim 30$ s following each evoked peak in CBF, there emerged slow oscillations in flow at a frequency of roughly 0.1 to $0.3 \mathrm{~Hz}$. Such low-frequency oscillations have been reported both as spontaneous fluctuations in ongoing flow ${ }^{37-39}$ as well as following functional activation. ${ }^{40}$ These emerging oscillations introduce additional waveform peaks that cannot be effectively fit using the standard, two-gamma function model. As an alternative approach for quantifying these new components of the $\mathrm{CBF}$ response, we performed time-frequency spectral analysis to 
(a)

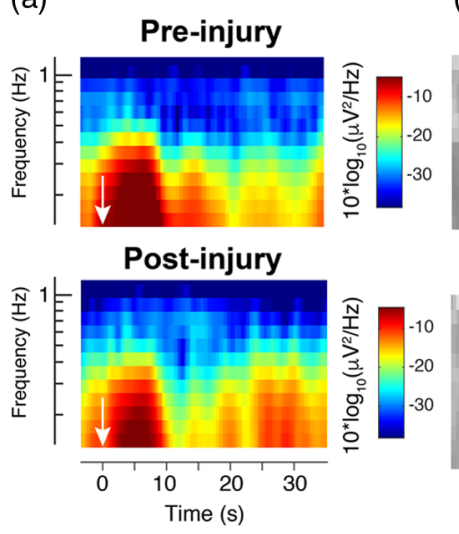

(b)

0 - 5 min

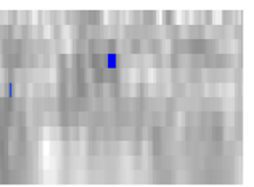

$15-20 \mathrm{~min}$

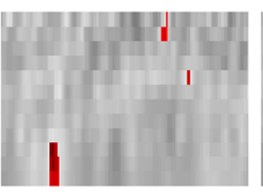

$5-10 \min$

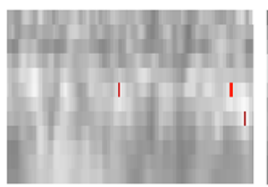

$20-25 \min$

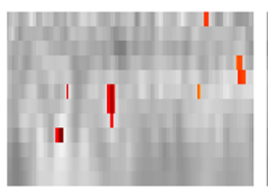

$10-15 \min$

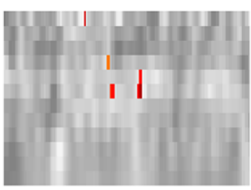

$25-30 \min$

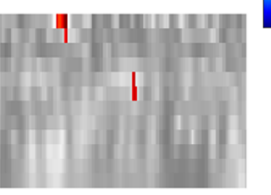

(c)

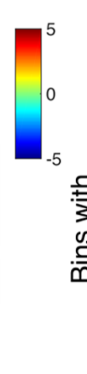

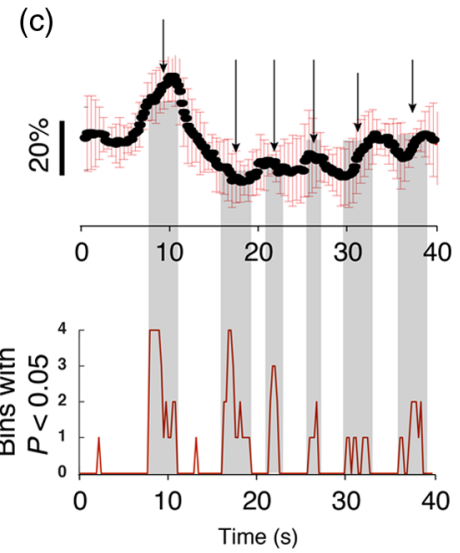

Fig. 9 Time-frequency analysis reveals significant changes in spectrogram after injury. (a) Representative time-frequency spectrograms from a single animal showing the alterations in spectrotemporal content following $\mathrm{CCl}$. The white arrows indicate the time point at which forelimb stimulation was applied. (b) Spectrotemporal changes over time, averaged over all animals. Each image depicts, in grayscale, the difference in spectrotemporal content via subtraction of the preinjury spectrogram (darker shades indicate decreases, brighter shades indicate increases). Superimposed in color on the grayscale difference spectrogram are points that, when analyzed over all experiments, demonstrated a difference from the preinjury values with $P<0.05$ using a two-tailed student's $t$-test. The colors are defined as in the colorbar of (a), and note that nearly all of the spectrotemporal power changes are focal increases. (c) Comparison of a representative postinjury $\triangle \mathrm{CBF}$ waveform (here, at a latency of $25 \mathrm{~min}$ following injury) with a plot showing the sum, over all experiments, of pixels showing alterations from baseline with $P<0.05$. Note that the significantly changed spectrotemporal features lined up with the cycles of apparent "oscillations" following injury. The gray shading projected onto the postinjury $\Delta C B F$ waveform (a representative plot) highlights temporal periods that contained statistically significant alterations in spectral power, assessed over all experiments $(n=10)$.

explore and more directly visualize these effects. While not typically applied to evoked responses, such spectral analysis has been used to explore dynamic, low-frequency aspects of ongoing cerebral autoregulation ${ }^{41}$ and provides an approach for characterizing the putative oscillatory behavior. As shown in Fig. 9, injury elicited changes in spectral power throughout the duration of the major peaks (increase and undershoot) of the CBF response, as well as in the interim between stimulus sets. Although the apparent spectrotemporal changes following injury resemble the development of temporally separated "notches" in the spectral power for frequencies below $1 \mathrm{~Hz}$, we explored whether these changes were significant across animals by assessing the patterns of spectrotemporal differences from preinjury values [Fig. 9(b)]. The regions of statistically significant spectrotemporal changes in power are relatively sparse; however, when summed over frequency and for all experiments, significant changes coincided with individual peaks in the "oscillatory" region, indicating a degree of phase locking [Fig. 9(c)].

To quantify this apparent coherence effect, we explored the ITC (Fig. 10), which can reveal spectrotemporal phase locking and variability among evoked responses. ${ }^{35}$ Similar to the results of spectral power analysis shown in Fig. 9, injury qualitatively changed ITC most prominently for regions following the initial CBF peak. A large fraction of the alterations in ITC were increases, most prominently in the period after the CBF undershoot and for frequencies above $0.4 \mathrm{~Hz}$. The one region that displayed a significant decrease in ITC was that corresponding to the spectrotemporal representation of the CBF undershoot, indicating greater variability of that portion of the HRF after injury. Although significant spectrotemporal increases in ITC $(P<0.05$ via two-tailed student's t-test) were apparent throughout the time course of the $\mathrm{CBF}$ response, we investigated the temporal distribution of ITC alterations [Fig. 10(c)] by analyzing flow as a series of three "regions" defined by physiologically relevant landmarks. When summed over frequencies, the period following the $\triangle \mathrm{CBF}$ undershoot (region three) demonstrated significant alterations from baseline.

\subsection{Correlations in the Dynamics of Cerebral Blood Flow and Electrophysiological Responses Following TBI}

Given the diversity of signals that were sensitive to injury, we sought to merge the findings by exploring correlations among their recovery kinetics. The matrix in Fig. 11 presents correlation coefficients comparing the post-CCI quantitative dynamics shown in Figs. 4 and 5. Overall, SSEP peak amplitudes and latencies were highly intercorrelated. In comparison, the recovery kinetics of the $\mathrm{CBF}$ components was relatively weakly intercorrelated. Among the cross-correlation terms indicative of coupling between hemodynamic and neural activity, we identified two major trends that are highlighted in Fig. 11. Notably, of all time-domain electrophysiological features that we explored, the amplitude of the CBF peak was most significantly correlated with the amplitude of P2 ( $z$-score $>3$, based on the SD of the distribution of Pearson's correlation coefficients). Additionally, while P2 represented the strongest cross-modal correlation in terms of amplitude, the latency of the initial $\triangle \mathrm{CBF}$ peak was highly correlated with all aspects of the SSEP peaks.

\section{Discussion}

A major repercussion of traumatic injury in the brain is an ensuing mismatch between oxygen supply and neural metabolic demand. $^{42,43}$ Changes in CBF and local tissue oxygenation dynamics ultimately reflect an interplay between CBF and 
(a)

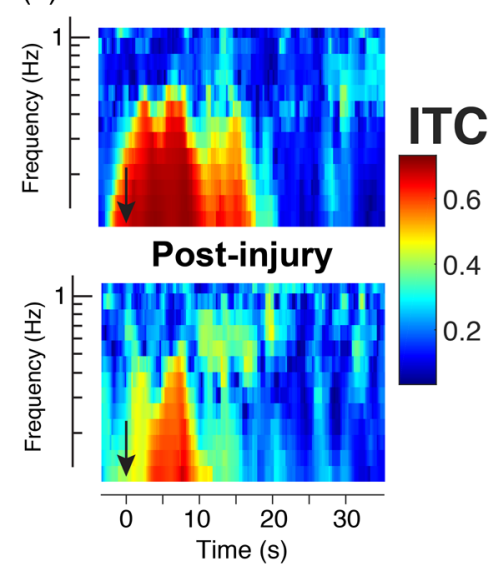

(b)

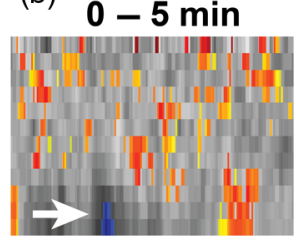

$15-20 \min$

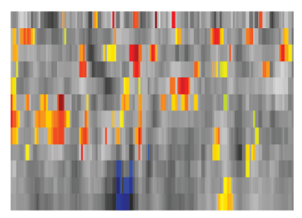

$5-10 \min$

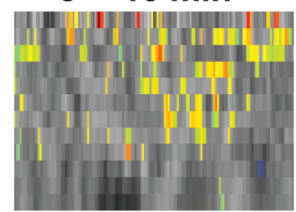

$20-25 \min$

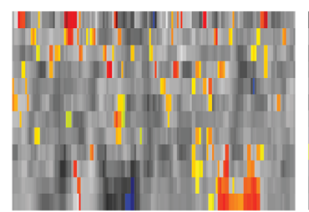

$10-15 \min$

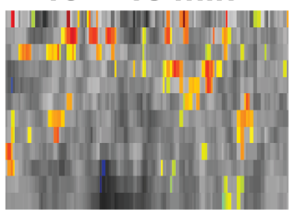

$25-30 \mathrm{~min}$

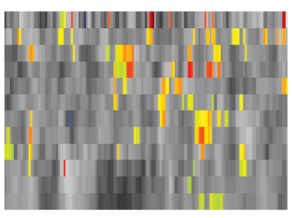

(c)
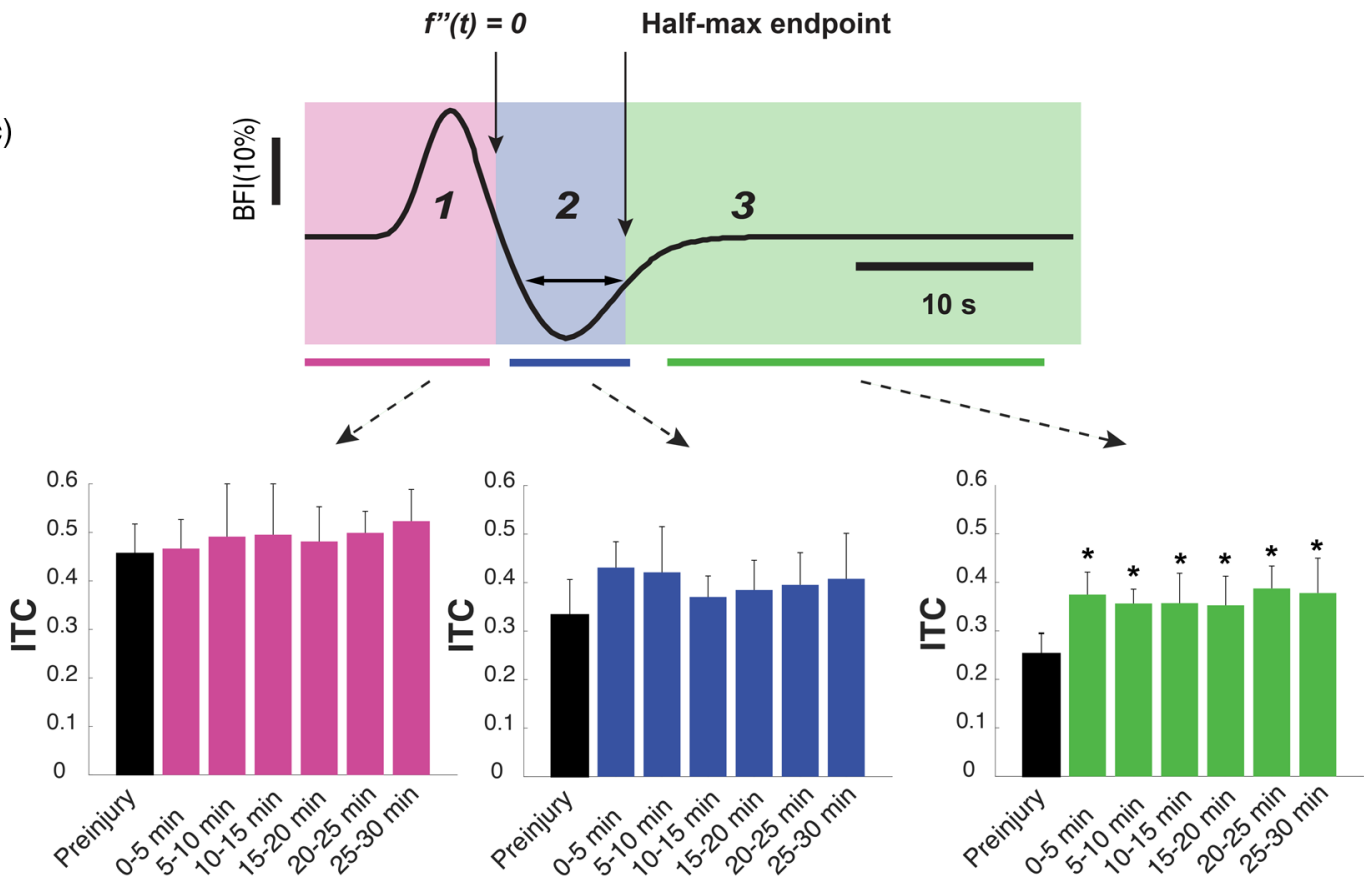

Fig. 10 ITC alterations following controlled cortical injury. (a) Representative spectrograms depicting the absolute magnitude of ITC before (top) and after (bottom) injury. These particular spectrograms are derived from the same animal as in Fig. 9(a). The color definitions are as indicated in the ITC colorbar; a value of 1 represents perfect, in-phase spectral responses. While the depicted values are real values, they represent the absolute magnitude of complex values that originally contain phase information. The black arrows indicate the time point at which forelimb stimulation was applied. (b) A series of ITC spectrograms, averaged over all animals, showing features that differ significantly from preinjury with $P<0.05$ obtained from a two-tailed student's t-test. As in Fig. 9(b), the statistically significant pixels, which are color-coded according to the colorbar of (a), are superimposed on a grayscale spectrogram representing the postinjury ITC spectrogram from which preinjury spectrogram values have been subtracted. While most of the changes represent increases in ITC at time points following the initial $\triangle \mathrm{CBF}$ waveform peak and undershoot, there was a significant decrease in ITC in the region of the undershoot itself, roughly the area of frequencies below $0.4 \mathrm{~Hz}$ between 12- and 16-s poststimulus (indicated by the white arrow in the top left superimposed image). (c) Regional assessment of ITC changes following injury. A sample $\triangle \mathrm{CBF}$ waveform fit divided into three regions for assessing changes in ITC at particular phases of the functional blood flow response is shown on top. The specific regions were defined as (1) an initial "rise" extending from the time directly after sensory stimulation cessation up through the point where the second derivative of the $\triangle \mathrm{CBF}$ fit passes zero; (2) the undershoot period, extending from the end of (1) through the latest point of intersection of the $\triangle C B F$ fit with the undershoot halfmaximum (or minimum, here) line; (3) the period of time following the main two phases of the $\triangle \mathrm{CBF}$ waveform and extending until the subsequent stimulus period. Asterisks in the right-most bar chart on the bottom indicate values that differ from baseline, preinjury values with $P<0.05$ according to a two-tailed Student's t-test. 


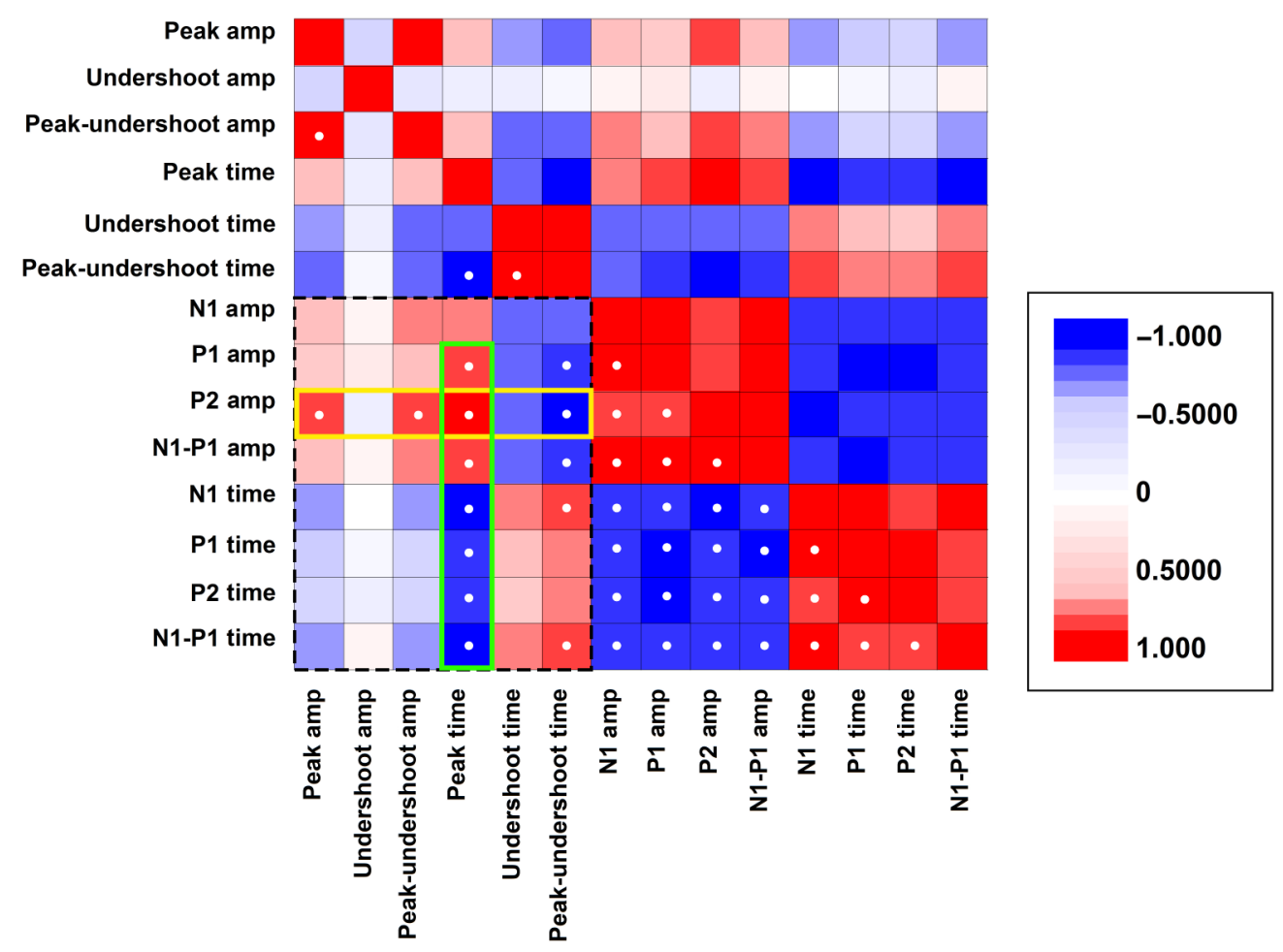

Fig. 11 Correlation matrix comparing the post-CCl recovery trends for the prominent features of the CBF and SSEP responses. Each matrix element represents the Pearson's correlation coefficient obtained by comparing the post- $\mathrm{CCl}$ time course (as in Figs. 4 and 5) of the major peaks and latencies. A correlation coefficient value of 1 represents identical waveforms, -1 indicates that the two traces are perfectly anticorrelated. The matrix is symmetrical about the diagonal. Correlation coefficients with an absolute value higher than 0.8 are indicated with white dots. The dotted rectangular perimeter delimits the matrix region representing correlation between the two modalities, optical and electrophysiological. The yellow perimeter highlights the high correlation between the $\mathrm{P} 2$ amplitude and postinjury hemodynamics, whereas the green perimeter highlights the fact that the $\triangle \mathrm{CBF}$ peak latency is highly correlated with all aspects of SSEPS.

changes in the cerebral metabolic rate of oxygen $\left(\mathrm{CMRO}_{2}\right)$, both of which are susceptible to alteration following acute injury. The primary mechanical injury, for example, can alter the vascular network that delivers oxygen to the parenchyma and, simultaneously, can alter neural activity and metabolic rate through a wide spectrum of mechanisms, including mechanical shearing ${ }^{44}$ or excitotoxicity due to damaged glia. ${ }^{45}$ Our concomitant measurements of $\mathrm{CBF}$ and electrical activity provide a picture of the repercussions of injury on both "supply and demand" aspects of the networks underlying sensation. While it is possible for injury to globally affect brain oxygenation (and, indeed, systemic physiology) as a result of compression of cardiorespiratory brainstem nuclei, our histological assessment does not find evidence of this scenario, and the changes in physiological signals likely reflect more subtle, transient perturbations.

While the general trends in SSEP alterations following injury are consistent with previous work in animal models, ${ }^{23,25,26,46}$ in the present study, we were able to identify time-domain alterations in the SSEP in finer detail. The most prominent SSEP peaks-N1, P1, and N2-exhibited differing alterations after injury, some of which are consistent with the known anatomical correlates of time-domain waveform. For instance, considering that the site of primary injury in CCI is the cortical gray matter, ${ }^{47}$ it is not entirely surprising that P1 ( 13-ms onset delay preinjury) exhibited the mildest decrease following injury given the fact that this component of the SSEP reflects subcortical activity related to thalamocortical relay. ${ }^{48}$ Likewise, the high sensitivity of P2 likely reflects the fact that its underlying generators are derived from cortico-cortical processing, which is sensitive to lateral cortical perturbations. For example, modulating cortical activity with topical application of pharmacological agents generally affects $\mathrm{N} 1$, but largely spares $\mathrm{P} 1 .{ }^{49}$ Consistent with this, in our previous work using a high-intensity focused ultrasound model for explosive blast, the mechanical insult penetrated deeper beneath the surface of the cortex and P1 was more significantly altered. ${ }^{23}$ The high correlation between hemodynamics and P2 may also reflect an intrinsic, disproportionately large influence of superficial cortical injury on electrical activity and hemodynamics within the cortical microvasculature. The superficial layers of the cortex feature extensive lateral networking with other cortical areas; ${ }^{50}$ thus, local disruption due to impact injury would likely have diffuse and far-reaching repercussions.

Within the temporal span of individual hemodynamic responses, the major features of the preinjury $\triangle \mathrm{CBF}$ that we observed-including both an initial peak and subsequent undershoot-have been observed in other DCS studies ${ }^{51,52}$ as well as studies using laser speckle imaging. ${ }^{17}$ While evidence of a significant undershoot is lacking in laser Doppler measurements, a clear inflection point between the CBF peak and return to baseline has been observed. ${ }^{53}$ A significant undershoot has also been reported in some fMRI studies, measured through arterial spin 
labeling, ${ }^{54-56}$ but not all. ${ }^{57}$ A major contributing factor underlying discrepancies in CBF results is likely the anatomical specificity of the measurement modality. For example, DCS measurements and speckle flow imaging are highly sensitive to flow in smaller vessels, whereas laser Doppler, which yields a minimal undershoot, is biased toward signals in large vessels, which generally exhibit smaller fractional changes in flow during functional activation.

The initial CBF peak reflects the influx of blood flow recruited by local changes in neural activity, ${ }^{58}$ and, on the timescale of days, its amplitude has been found to be reduced following traumatic injury ${ }^{59}$ and stroke. ${ }^{60}$ To our knowledge, however, the impact of injury on sensory-evoked CBF, both in terms of amplitude and temporal waveform, has not been explored within the first moments following TBI. It remains to be seen whether our observed acute dynamics, which depict significant recovery of some aspects of the sensory-evoked $\triangle \mathrm{CBF}$, continuously evolve into the previously reported longterm deficits. In terms of injury-induced alterations in peak CBF timing, the same prior investigations reported a delayed flow onset that is observable on the timescale of days following injury. Our observation of a reduced onset latency is innovative; however, it may be unique to the immediate aftermath of acute injury. In general, the fact that most aspects of the $\triangle \mathrm{CBF}$ response and SSEPs substantially recover within a relatively short period of time is remarkable given the known chronic effects that TBI exerts on baseline hemodynamics, notably, changes in cerebrovascular autoregulation. ${ }^{61,62}$ The findings concur with previous reports that functional $\mathrm{CBF}$ is independent of baseline values, ${ }^{.3,63,64}$ however, our results indicate that this independence extends to injury-induced global hemodynamic changes.

It has long been assumed that the sensory-evoked hemodynamic response undershoot is primarily a vascular phenomenon, representing a poststimulus period of increased cerebral blood volume even after $\mathrm{CBF}$ and $\mathrm{CMRO}_{2}$ have returned to baseline ${ }^{65}$ However, more recent work strongly indicates that the poststimulus undershoot is highly dependent on neural activity ${ }^{56}$ Our results depict the postinjury undershoot as chronically attenuated and more variable. Similarly, SSEP components also displayed a marked increase in variability in both time and frequency domain. These observations are, therefore, consistent with the hypothesis that neural activity plays a significant role in shaping the undershoot. However, the fact that the variability and amplitude of the undershoot does not recover as fully as other CBF waveform components even after SSEP attributes have returned to baseline status suggests that the prolonged undershoot alterations may not be primarily shaped by neural activity. Future studies involving systematic variation of stimulus parameters may be able to more directly probe this hypothesis.

Using a variety of techniques, low-frequency $(<0.5 \mathrm{~Hz}) \mathrm{CBF}$ oscillations similar to the ones we detected following injury (Figs. 9 and 10) have been previously observed, both spontaneously and following stimulus-evoked CBF, in the form of vasomotion..$^{37,40,66,67}$ While the physiological basis of these oscillations is still currently unclear, multiple mechanisms have been suggested, including emergent properties of molecular signaling pathways ${ }^{68}$ or more fundamental fluid mechanical principles. ${ }^{69,70}$ Such fluid mechanical models derive an oscillatory component based on inclusion of an inertial term, owing to blood volume in larger, more distal venules and arterioles. ${ }^{71}$ A simplistic, general interpretation of the results is that the emergence of postundershoot oscillations in CBF reflects perturbation of an intrinsically oscillatory system, regardless of whether the affected components are mechanical or phenomenological (e.g., related to molecular signaling).

In emergency medicine, there is a well-known principle of the "golden hour," the period of time following a traumatic injury within which medical intervention can have a maximal impact on patient outcome. ${ }^{72}$ Although the precise duration is debated, ${ }^{73,74}$ timely transport to a hospital is essential. Our experimental investigation focused on disruptions in neurovascular coupling that occurs within this relatively short time window, and we identified several metrics that were profoundly altered by TBI. It is, however, both possible and likely that at later time points, the trends we observed will change or reverse. The rapid energy depletion associated with disrupted $\mathrm{CBF}$ and mechanical shearing of neurons dramatically increases extracellular $\mathrm{K}^{+}$and glutamate concentrations. ${ }^{75,76}$ In turn, resulting excitotoxicity triggers a variety of rapidly evolving adverse events through the "ischemic cascade." For example, initial cytotoxic edema is followed by the onset of vasogenic edema within the first few hours. ${ }^{77,78}$ These various sequelae will likely be reflected in the optical and electrophysiological signals. Future experimental work focusing on the longerterm evolution of neurovascular coupling following CCI could provide a fuller physiological context for the initial effects that we characterized in this present study. On a technical level, focusing on physiological signals during the early timeframe of injury provided a way to mitigate potential confounds owing to anesthesia pharmacodynamics, which could be affected by injury.

A general challenge for parsing the bulk signals obtained through diffuse optical neuromonitoring techniques is that the spatial resolution is limited because the detected signals include light that has traversed a large span of optical paths in tissue. ${ }^{79}$ In the case of DCS, although the signal origin is putatively weighted toward microvasculature, the fidelity with which the bulk signal aligns with a microscale portrait of blood flow in the layers of the cerebral cortex remains to be quantified. Future work in animal models of TBI that supplements DCS measurements with high-resolution imaging of activity in microvasculature, such as optical coherence angiography ${ }^{80,81}$ or two-photon microscopy, ${ }^{82}$ may better inform interpretation.

\section{Conclusion}

While significant progress has been made toward understanding the repercussions and mechanisms of mTBI at the cellular level using invasive, high-resolution techniques, biomarkers for rapidly assessing human subjects in active settings outside the clinic are constrained to signals that can be obtained with noninvasive technology. Such measurement modalities, however, are limited in their ability to spatially resolve signals and biomarkers observed in preclinical work. As we have demonstrated, functional neurovascular coupling is a property that can be measured with low-resolution techniques and contains information about microscale physiological function. This information can be extracted from variable, high-noise physiological recordings because they are temporally synchronized with external stimuli that can be delivered in a controlled manner. The event-related optical and electrophysiological changes that we have discovered, albeit correlative, are significantly indicative of TBI in an animal model. Notably, the dynamic alterations we observed in stimulus-evoked blood flow and electrophysiological 
responses were not associated with significant tissue damage; rather, our histological examination revealed only a small increase in astrogliosis associated with CCI. We expect that these functional indicators will ultimately add informative dimensions to current multimodal approaches for diagnosing mTBI in human subjects, given the heterogeneity of baseline values across populations.

\section{Disclosures}

The authors report no financial interests or potential conflicts of interest related to this work.

\section{Acknowledgments}

The mention of commercial products, their sources, or their use in connection with material reported herein is not to be construed as either an actual or implied endorsement of such products by the Department of Health and Human Services. The authors thank Dr. A. Parthasarathy, Dr. W. Baker, Dr. T. Durduran, Dr. A. G. Yodh, Dr. G. Yu, Dr. D. Busch, Dr. A. Koller, Dr. D. Ress, and Dr. J. Pfefer for helpful discussions, and Dr. S. Vasudevan for technical assistance. The work was supported by the National Science Foundation (Award Nos. 1541612 and 1641133) as well as intramural recruitment funds from New York Medical College.

\section{References}

1. M. Faul et al., Traumatic Brain Injury in the United States: Emergency Department Visits, Hospitalizations, and Deaths, Centers for Disease Control and Prevention, National Center for Injury Prevention and Control, Atlanta, Georgia (2010).

2. A. I. Maas, N. Stocchetti, and R. Bullock, "Moderate and severe traumatic brain injury in adults," Lancet Neurol. 7, 728-741 (2008).

3. C. Eierud et al., "Neuroimaging after mild traumatic brain injury: review and meta-analysis," NeuroImage Clin. 4, 283-294 (2014).

4. D. Gronwall and P. Wrightson, "Cumulative effect of concussion," Lancet 306, 995-997 (1975).

5. M. Gaetz, D. Goodman, and H. Weinberg, "Electrophysiological evidence for the cumulative effects of concussion," Brain Inj. 14, 1077-1088 (2000).

6. K. M. Guskiewicz et al., "Cumulative effects associated with recurrent concussion in collegiate football players: the NCAA concussion study," J. Am. Med. Assoc. 290, 2549-2555 (2003).

7. D. S. Alwis et al., "Sensory cortex underpinnings of traumatic brain injury deficits," PLoS One 7, e52169 (2012).

8. F. J. Gallun et al., "Implications of blast exposure for central auditory function: a review," J. Rehabil. Res. Dev. 49, 1059-1074 (2012).

9. G. C. Cockerham et al., "Eye and visual function in traumatic brain injury," J. Rehabil. Res. Dev. 46, 811-818 (2009).

10. K. D. Brahm et al., "Visual impairment and dysfunction in combatinjured service members with traumatic brain injury," Optom. Vision Sci. 86, 817-825 (2009).

11. C. D. Callahan and J. H. Hinkebein, "Assessment of anosmia after traumatic brain injury: performance characteristics of the University of Pennsylvania smell identification test," J. Head Trauma Rehabil. 17, 251-256 (2002).

12. A. Irimia et al., "Electroencephalographic inverse localization of brain activity in acute traumatic brain injury as a guide to surgery, monitoring and treatment," Clin. Neurol. Neurosurg. 115, 2159-2165 (2013).

13. C. Lecrux and E. Hamel, "The neurovascular unit in brain function and disease," Acta Physiol. 203, 47-59 (2011).

14. N. Nishimura et al., "Limitations of collateral flow after occlusion of a single cortical penetrating arteriole," J. Cereb. Blood Flow Metab. 30, 1914-1927 (2010).

15. G. J. Del Zoppo, "Microvascular changes during cerebral ischemia and reperfusion," Cerebrovasc. Brain Metab. Rev. 6, 47-96 (1994).
16. T. Durduran and A. G. Yodh, "Diffuse correlation spectroscopy for non-invasive, micro-vascular cerebral blood flow measurement," NeuroImage 85(1), 51-63 (2014).

17. T. Durduran et al., "Spatiotemporal quantification of cerebral blood flow during functional activation in rat somatosensory cortex using laser-speckle flowmetry," J. Cereb. Blood Flow Metab. 24, 518-525 (2004).

18. C. Zhou et al., "Diffuse optical correlation tomography of cerebral blood flow during cortical spreading depression in rat brain," Opt. Express 14, 1125 (2006).

19. C. Zhou et al., "Diffuse optical monitoring of hemodynamic changes in piglet brain with closed head injury," J. Biomed. Opt. 14, 034015 (2009).

20. Y. Shitaka et al., "Repetitive closed-skull traumatic brain injury in mice causes persistent multifocal axonal injury and microglial reactivity," J. Neuropathol. Exp. Neurol. 70, 551-567 (2011).

21. B. Mouzon et al., "Repetitive mild traumatic brain injury in a mouse model produces learning and memory deficits accompanied by histological changes," J. Neurotrauma 29, 2761-2773 (2012).

22. Y. Xiong, A. Mahmood, and M. Chopp, "Animal models of traumatic brain injury," Nat. Rev. Neurosci. 14, 128-142 (2013).

23. J. Fisher et al., "Real-time detection and monitoring of acute brain injury utilizing evoked electroencephalographic potentials," IEEE Trans. Neural Syst. Rehabil. Eng.. 24(9), 1003-1012 (2016).

24. S. Huang et al., "Epidermal electrode technology for detecting ultrasonic perturbation of sensory brain activity," IEEE Trans. Biomed. Eng. (2017) (in press).

25. K. Sakatani, H. Iizuka, and W. Young, "Somatosensory evoked potentials in rat cerebral cortex before and after middle cerebral artery occlusion," Stroke 21, 124-132 (1990).

26. D. Wu et al., "Study of the origin of short- and long-latency SSEP during recovery from brain ischemia in a rat model," Neurosci. Lett. $\mathbf{4 8 5}$, 157-161 (2010).

27. D. L. Brody et al., "Electromagnetic controlled cortical impact device for precise, graded experimental traumatic brain injury," J. Neurotrauma 24, 657-673 (2007).

28. C. S. Budinich et al., "Mouse brain PSA-NCAM levels are altered by graded-controlled cortical impact injury," Neural Plast. 2012, 378307 (2012).

29. P. M. Washington et al., "The effect of injury severity on behavior: a phenotypic study of cognitive and emotional deficits after mild, moderate, and severe controlled cortical impact injury in mice," J. Neurotrauma 29, 2283-2296 (2012).

30. J.-H. Yi et al., "Alterations in sulfated chondroitin glycosaminoglycans following controlled cortical impact injury in mice," J. Comp. Neurol. 520, 3295-3313 (2012).

31. T. Durduran et al., "Diffuse optics for tissue monitoring and tomography," Rep. Prog. Phys. 73(7), 076701 (2010).

32. Correlator.com, Las Vegas, Nevada, www.correlator.com.

33. G. Paxinos and K. B. J. Franklin, The Mouse Brain in Stereotaxic Coordinates, Gulf Professional Publishing, Oxford, United Kingdom (2004).

34. M. A. Lindquist et al., "Modeling the hemodynamic response function in fMRI: efficiency, bias and mis-modeling," NeuroImage 45, S187S198 (2009).

35. A. Delorme and S. Makeig, "EEGLAB: an open source toolbox for analysis of single-trial EEG dynamics including independent component analysis," J. Neurosci. Methods 134, 9-21 (2004).

36. E. S. Lein et al., "Genome-wide atlas of gene expression in the adult mouse brain," Nature 445, 168-176 (2007).

37. J. E. W. Mayhew et al., "Cerebral vasomotion: a $0.1-\mathrm{Hz}$ oscillation in reflected light imaging of neural activity," NeuroImage 4, 183-193 (1996).

38. R. R. Diehl et al., "Spontaneous oscillations in cerebral blood flow velocity in normal humans and in patients with carotid artery disease," Neurosci. Lett. 127, 5-8 (1991).

39. U. Dirnagl et al., "Continuous measurement of cerebral cortical blood flow by laser-Doppler flowmetry in a rat stroke model,' J. Cereb. Blood Flow Metab. 9, 589-596 (1989).

40. D. Kleinfeld et al., "Fluctuations and stimulus-induced changes in blood flow observed in individual capillaries in layers 2 through 4 of rat neocortex," Proc. Natl. Acad. Sci. U. S. A. 95, 15741-15746 (1998). 
41. M. M. Placek et al., "Applying time-frequency analysis to assess cerebral autoregulation during hypercapnia," PLoS One 12, e0181851 (2017).

42. C. Werner and K. Engelhard, "Pathophysiology of traumatic brain injury," Br. J. Anaesth. 99, 4-9 (2007).

43. P. Toth et al., "Traumatic brain injury-induced autoregulatory dysfunction and spreading depression-related neurovascular uncoupling: pathomechanisms, perspectives, and therapeutic implications," Am. J. Physiol.-Heart Circ. Physiol. 311, H1118-H1131 (2016).

44. W. D. Dietrich, O. Alonso, and M. Halley, "Early microvascular and neuronal consequences of traumatic brain injury: a light and electron microscopic study in rats," J. Neurotrauma 11, 289-301 (1994).

45. J.-H. Yi and A. S. Hazell, "Excitotoxic mechanisms and the role of astrocytic glutamate transporters in traumatic brain injury," Neurochem. Int. 48, 394-403 (2006).

46. Y. Ma et al., "Time jitter of somatosensory evoked potentials in recovery from hypoxic-ischemic brain injury," J. Neurosci. Methods 201, 355360 (2011).

47. Y. Chen et al., "A modified controlled cortical impact technique to model mild traumatic brain injury mechanics in mice," Front. Neurol. 5, 100 (2014).

48. M. R. Nuwer, "Fundamentals of evoked potentials and common clinical applications today," Electroencephalogr. Clin. Neurophysiol. 106, 142 148 (1998).

49. R. J. Staba, P. C. Bergmann, and D. S. Barth, "Dissociation of slow waves and fast oscillations above $200 \mathrm{~Hz}$ during GABA application in rat somatosensory cortex," J. Physiol. 561, 205-214 (2004).

50. V. B. Mountcastle, Perceptual Neuroscience: The Cerebral Cortex, Harvard University Press, Cambridge, Massachusetts (1998).

51. T. Durduran et al., "Diffuse optical measurement of blood flow, blood oxygenation, and metabolism in a human brain during sensorimotor cortex activation," Opt. Lett. 29, 1766-1768 (2004).

52. N. Roche-Labarbe et al., "Somatosensory evoked changes in cerebral oxygen consumption measured non-invasively in premature neonates," NeuroImage 85(1), 279-286 (2014).

53. B. M. Ances et al., "Coupling of neural activation to blood flow in the somatosensory cortex of rats is time-intensity separable, but not linear," J. Cereb. Blood Flow Metab. 20, 921-930 (2000).

54. K. Uludağ et al., "Coupling of cerebral blood flow and oxygen consumption during physiological activation and deactivation measured with fMRI," Neurolmage 23, 148-155 (2004).

55. O. Leontiev et al., "Coupling of cerebral blood flow and oxygen metabolism is conserved for chromatic and luminance stimuli in human visual cortex," Neurolmage 68, 221-228 (2013).

56. K. J. Mullinger et al., "Poststimulus undershoots in cerebral blood flow and BOLD fMRI responses are modulated by poststimulus neuronal activity," Proc. Natl. Acad. Sci. U. S. A. 110, 13636-13641 (2013).

57. R. D. Hoge et al., "Stimulus-dependent BOLD and perfusion dynamics in human V1," NeuroImage 9, 573-585 (1999).

58. R. B. Buxton, E. C. Wong, and L. R. Frank, "Dynamics of blood flow and oxygenation changes during brain activation: the balloon model," Magn. Reson. Med. 39, 855-864 (1998).

59. J.-P. Niskanen et al., "Monitoring functional impairment and recovery after traumatic brain injury in rats by fMRI," J. Neurotrauma 30, 546556 (2013).

60. M. D’Esposito, L. Y. Deouell, and A. Gazzaley, "Alterations in the BOLD fMRI signal with ageing and disease: a challenge for neuroimaging," Nat. Rev. Neurosci. 4, 863-872 (2003).

61. W. Lewelt, L. W. Jenkins, and J. D. Miller, "Autoregulation of cerebral blood flow after experimental fluid percussion injury of the brain," J. Neurosurg. 53, 500-511 (1980).

62. K. Engelborghs et al., "Impaired autoregulation of cerebral blood flow in an experimental model of traumatic brain injury," J. Neurotrauma 17, 667-677 (2000).

63. T.-Q. Li, M. E. Moseley, and G. Glover, "A FAIR study of motor cortex activation under normo- and hypercapnia induced by breath challenge," NeuroImage 10, 562-569 (1999).

64. T.-Q. Li et al., "Changes in baseline cerebral blood flow in humans do not influence regional cerebral blood flow response to photic stimulation," J. Magn. Reson. Imaging 12, 757-762 (2000).

65. R. B. Buxton et al., "Modeling the hemodynamic response to brain activation," NeuroImage 23(Suppl. 1), S220-S233 (2004).
66. H. Obrig et al., "Spontaneous low frequency oscillations of cerebral hemodynamics and metabolism in human adults," NeuroImage 12, 623-639 (2000)

67. T. Katura et al., "Quantitative evaluation of interrelations between spontaneous low-frequency oscillations in cerebral hemodynamics and systemic cardiovascular dynamics," NeuroImage 31, 1592-1600 (2006).

68. Y. Itoh and N. Suzuki, "Control of brain capillary blood flow," J. Cereb. Blood Flow Metab. 32, 1167-1176 (2012).

69. J. H. Kim and D. Ress, "Arterial impulse model for the BOLD response to brief neural activation," Neurolmage 124, 394-408 (2016).

70. J. H. Kim et al., "Model of the transient neurovascular response based on prompt arterial dilation," J. Cereb. Blood Flow Metab. 33, 14291439 (2013).

71. D. Ress et al., "A model for transient oxygen delivery in cerebral cortex," Front. Neuroenerg. 1, 3 (2009).

72. R. Ruff, "Two decades of advances in understanding of mild traumatic brain injury," J. Head Trauma Rehabil. 20, 5-18 (2005).

73. M. M. Dinh et al., "Redefining the golden hour for severe head injury in an urban setting: the effect of prehospital arrival times on patient outcomes," Injury 44, 606-610 (2013).

74. E. B. Lerner and R. M. Moscati, "The golden hour: scientific fact or medical 'urban legend'?" Acad. Emerg. Med. 8, 758-760 (2001).

75. Y. Katayama et al., "Massive increases in extracellular potassium and the indiscriminate release of glutamate following concussive brain injury," J. Neurosurg. 73, 889-900 (1990).

76. P. Nilsson et al., "Changes in cortical extracellular levels of energyrelated metabolites and amino acids following concussive brain injury in rats," J. Cereb. Blood Flow Metab. 10, 631-637 (1990).

77. S. Nag, J. L. Manias, and D. J. Stewart, "Pathology and new players in the pathogenesis of brain edema," Acta Neuropathol. 118, 197-217 (2009).

78. J. J. Donkin and R. Vink, "Mechanisms of cerebral edema in traumatic brain injury: therapeutic developments," Curr. Opin. Neurol. 23, 293299 (2010).

79. D. A. Boas, A. M. Dale, and M. A. Franceschini, "Diffuse optical imaging of brain activation: approaches to optimizing image sensitivity, resolution, and accuracy," NeuroImage 23(Suppl. 1), S275-S288 (2004).

80. V. J. Srinivasan et al., "Rapid volumetric angiography of cortical microvasculature with optical coherence tomography," Opt. Lett. 35, 43-45 (2010).

81. M. S. Mahmud et al., "Review of speckle and phase variance optical coherence tomography to visualize microvascular networks," $J$. Biomed. Opt. 18, 050901 (2013).

82. A. Y. Shih et al., "Two-photon microscopy as a tool to study blood flow and neurovascular coupling in the rodent brain," J. Cereb. Blood Flow Metab. 32, 1277-1309 (2012).

Hyounguk Jang received his $\mathrm{BS}$ degree and $\mathrm{PhD}$ in physics from the University of Sungkyunkwan, Korea, in 2002 and Kansas State University in 2010, respectively. He performed postdoctoral research in the Department of Physics, University of Maryland, from 2013 and the Department of Bioengineering, University of Maryland, from 2015. Currently, he is a postdoctoral researcher in the Department of Physiology, New York Medical College, and a scholar-in-residence at the U.S. Food and Drug Administration (FDA).

Stanley Huang received his BS and MS degrees in biomedical engineering from Johns Hopkins University, Baltimore, Maryland, in 2003 and 2005, respectively. He received his $\mathrm{PhD}$ in biomedical engineering from Columbia University, New York City in 2011. Currently, he is a research fellow at the U.S. FDA. His research interests include biosignal processing, bioelectronics, medical imaging, and neurological devices.

Daniel X. Hammer has over 20 years of experience in the invention, development, and application of biomedical optics to a broad range of interdisciplinary research programs. His current research interests include optical coherence tomography, adaptive optics, and neuroimaging. $\mathrm{He}$ is currently the deputy director of the Division of Biomedical Physics (DBP) within the Center for Devices and Radiological Health's (CDRH) Office of Science and Engineering Laboratories (OSEL) at the U.S. Food and Drug Administration (FDA) in Silver Spring, Maryland. He is a member of SPIE (senior member), the Optical Society of America, the Association for 
Research in Vision and Ophthalmology, and the American Institute for Medical and Biological Engineering (fellow).

Lin Wang was a graduate student at New York Medical College and is currently a medical student at the Medical College of Wisconsin. Her current research interests include improving medical diagnostic tools and modalities, particularly for mild traumatic brain injury (TBI). She has also been involved in the design of a portable device for diagnosing and quantifying neurovascular alternations after mild TBI at New York Medical College.

Harmain Rafi received her BS degree in neuroscience from George Mason University, Fairfax, Virginia, in 2016. Her previous work includes retrospective research on spinal cord stimulators at INOVA Fairfax hospital and locomotor behavioral studies in rats at the Krasnow Institute. Currently, she is an ORISE fellow at the U. S. FDA in Silver Spring, Maryland, working in the Neural Implants Lab.

Meijun Ye received her PhD in neuroscience from the University of Arkansas for Medical Sciences. After that she did her postdoctoral research in visual sciences at Yale University. She joined the Neurological Devices Laboratory at the U.S. FDA in 2013. Her research interests include neurophysiological signatures of TBI, safety and effectiveness of neural diagnostic and intervention devices, cellular and circuitry mechanisms of brain injury, retinal neural circuits, and neural interactions in the reticular activating system.

Cristin G. Welle received her BS degree from the College of William and Mary in 2004 and her PhD from the University of Pennsylvania in 2010. She is an assistant professor at the University of Colorado, Denver. Previously, she was a staff scientist at the U.S. FDA, where she led the Neural Interfaces Laboratory. Her research interests center on using small animal models to explore long-term safety and performance of medical devices that interface with the nervous system.

Jonathan A. N. Fisher received his BA degree and $\mathrm{PhD}$ in physics from the University of Pennsylvania in 2000 and 2007, respectively. $\mathrm{He}$ performed his postdoctoral research in neuroscience at The Rockefeller University. From 2013 to 2014, he was an ORISE faculty fellow at the U.S. FDA/CDRH/OSEL/Division of Biomedical Physics. Currently, he is an assistant professor of physiology at New York Medical College and his awards include a Blavatnik Award for Young Scientists. 